\title{
Lifshitz spacetimes, solitons, and generalized BTZ black holes in quantum gravity at a Lifshitz point
}

\author{
Fu-Wen Shu, ${ }^{a, b}$ Kai Lin, ${ }^{a, c}$ Anzhong Wang ${ }^{a, d, 1}$ and Qiang $\mathbf{W u}{ }^{a}$ \\ ${ }^{a}$ Institute for Advanced Physics \& Mathematics, Zhejiang University of Technology, \\ Hangzhou 310032, China \\ ${ }^{b}$ Center for Relativistic Astrophysics and High Energy Physics, Nanchang University, \\ Nanchang 330031, China \\ ${ }^{c}$ Instituto de Física, Universidade de São Paulo, \\ CP 66318, 05315-970, São Paulo, Brazil \\ ${ }^{d}$ GCAP-CASPER, Physics Department, Baylor University, \\ Waco, TX 76798-7316, U.S.A. \\ E-mail: shufuwen@ncu.edu.cn, 1k314159@hotmail.com, \\ anzhong_wang@baylor.edu, wuq@zjut.edu.cn
}

ABSTRACT: In this paper, we study static vacuum solutions of quantum gravity at a fixed Lifshitz point in $(2+1)$ dimensions, and present all the diagonal solutions in closed forms in the infrared limit. The exact solutions represent spacetimes with very rich structures: they can represent generalized BTZ black holes, Lifshitz space-times or Lifshitz solitons, in which the spacetimes are free of any kind of space-time singularities, depending on the choices of the free parameters of the solutions. We also find several classes of exact static non-diagonal solutions, which represent similar space-time structures as those given in the diagonal case. The relevance of these solutions to the non-relativistic Lifshitz-type gauge/gravity duality is discussed.

KEYwords: AdS-CFT Correspondence, Black Holes, Spacetime Singularities, Holography and condensed matter physics (AdS/CMT)

ARXIV EPRINT: 1403.0946

\footnotetext{
${ }^{1}$ Corresponding author.
} 


\section{Contents}

1 Introduction 1

2 Non-projectable HL gravity 4

2.1 Field equations 5

$\begin{array}{lll}2.2 & \text { Ghost-free and stability conditions } & 6\end{array}$

3 Static vacuum solutions in the IR limit $\quad 7$

3.1 Lifshitz spacetime 8

3.2 Asymptotical Lifshitz spacetimes $\quad 9$

$\begin{array}{llr}3.2 .1 & s>1 & 12\end{array}$

$\begin{array}{lll}3.2 .2 & 0<s<1 & 15\end{array}$

$\begin{array}{ll}3.2 .3-1<s<0 & 18\end{array}$

$\begin{array}{ll}3.2 .4 & s<-1\end{array} 22$

3.3 Generalized BTZ black holes 23

3.4 Solutions with $s=-1 \quad 24$

$\begin{array}{ll}3.5 & \text { Solutions with } s=0\end{array}$

4 Static vacuum solutions for the non-diagonal case $N^{r} \neq 0 \quad 26$

$\begin{array}{ll}4.1 \text { Solutions with } \lambda=1 & 26\end{array}$

$\begin{array}{lll}4.1 .1 \beta=0 & 27\end{array}$

$4.1 .2 \beta \neq 0 \quad 28$

$\begin{array}{lll}4.2 & \text { Solutions with } \lambda \neq 1 & 29\end{array}$

$\begin{array}{lll}4.2 .1 W=0 & 29\end{array}$

$\begin{array}{lll}4.2 .2 W=z & 30\end{array}$

4.2.3 Solutions with $W \neq 0, z$ and $\beta=0 \quad 32$

4.2.4 Solutions with $W \neq 0, z$ and $\lambda=1 / 2$

5 Conclusions $\quad 33$

A Functions $F_{V}, F^{i j}$ and $F_{a}^{i j}$

\section{Introduction}

Anisotropic scaling plays a fundamental role in quantum phase transitions in condensed matter and ultracold atomic gases $[1,2]$. Recently, such studies have received considerable momenta from the community of string theory in the content of gauge/gravity duality [3-5]. This is a duality between a quantum field theory (QFT) in D-dimensions and a quantum gravity, such as string theory, in $(\mathrm{D}+1)$-dimensions. An initial example was 
found between the supersymmetric Yang-Mills gauge theory with maximal supersymmetry in four-dimensions and a string theory on a five-dimensional anti-de Sitter space-time in the low energy limit [6-8]. Soon, it was discovered that such a duality is not restricted to the above systems, and can be valid among various theories and in different spacetime backgrounds [3-5].

One of the remarkable features of the duality is that it relates a strong coupling QFT to a weak coupling gravitational theory, or vice versa. This is particular attractive to condensed matter physicists, as it may provide hopes to understand strong coupling systems encountered in quantum phase transitions, by simply studying the dual weakly coupling gravitational theory [9-12]. Otherwise, it has been found extremely difficult to study those systems. Such studies were initiated in [13], in which it was shown that nonrelativistic QFTs that describe multicritical points in certain magnetic materials and liquid crystals may be dual to certain nonrelativistic gravitational theories in the Lifshitz space-time background, ${ }^{1}$

$$
d s^{2}=-\left(\frac{r}{\ell}\right)^{2 z} d t^{2}+\left(\frac{r}{\ell}\right)^{2} d x^{i} d x^{i}+\left(\frac{\ell}{r}\right)^{2} d r^{2},
$$

where $z$ is a dynamical critical exponent, and $\ell$ a dimensional constant. Clearly, the above metric is invariant under the anisotropic scaling,

$$
t \rightarrow b^{z} t, \quad \mathbf{x} \rightarrow b \mathbf{x}
$$

provided that $r$ scales as $r \rightarrow b^{-1} r$. Thus, for $z \neq 1$ the relativistic scaling is broken, and to have the above Lifshitz space-time as a solution of general relativity (GR), it is necessary to introduce gauge fields to create a preferred direction, so that the anisotropic scaling (1.2) becomes possible. In [13], this was realized by two p-form gauge fields with $p=1,2$, and was soon generalized to different cases [17-20].

It should be noted that the Lifshitz space-time is singular at $r=0$ [13], and this singularity is generic in the sense that it cannot be eliminated by simply embedding it to high-dimensional spacetimes, and that test particles/strings become infinitely excited when passing through the singularity [21, 22]. To resolve this issue, various scenarios have been proposed [23-27]. There have been also attempts to cover the singularity by horizons [28-49], and replace it by Lifshitz solitons [50-52].

On the other hand, starting with the anisotropic scaling (1.2), recently Hořava constructed a theory of quantum gravity, the so-called Hořava-Lifshitz (HL) theory [53], which is power-counting renormalizable, and lately has attracted a great deal of attention, due to its remarkable features when applied to cosmology and astrophysics [54-57]. The HL theory is based on the perspective that Lorentz symmetry should appear as an emergent symmetry at long distances, but can be fundamentally absent at short ones [58, 59]. In the ultraviolet (UV), the system exhibits a strong anisotropic scaling between space and time with $z \geq D$, while at the infrared (IR), high-order curvature corrections become negligible,

\footnotetext{
${ }^{1}$ Another space-time that is conjectured to be holographically dual to such strongly coupled systems is the Schrödingier space-time [14-16], in which the related symmetry algebra is Schrödingier, instead of Lifshitz. However, to realize such an algebra, it was found that the space-time needs to be $(D+2)$-dimensions, instead of $(D+1)$-dimensions.
} 
and the lowest order terms $R$ and $\Lambda$ take over, whereby the Lorentz invariance (with $z=1$ ) is expected to be "accidentally restored," where $R$ denotes the D-dimensional Ricci scalar of the leaves $t=$ Constant, and $\Lambda$ the cosmological constant.

Since the anisotropic scaling (1.2) is built in by construction in the HL gravity, it is natural to expect that the HL gravity provides a minimal holographic dual for nonrelativistic Lifshitz-type field theories with the anisotropic scaling and dynamical exponent $z$. Indeed, recently it was showed that the Lifshitz spacetime (1.1) is a vacuum solution of the HL gravity in $(2+1)$ dimensions, and that the full structure of the $z=2$ anisotropic Weyl anomaly can be reproduced in dual field theories [60], while its minimal relativistic gravity counterpart yields only one of two independent central charges in the anomaly.

In this paper, we shall provide further evidence to support the above speculations, by constructing various solutions of the HL gravity, and show that these solutions provide all the space-time structures found recently in GR with various matter fields, including the Lifshitz solitons [50-52] and generalized BTZ black holes. Some solutions represent incomplete space-time, and extensions beyond certain horizons are needed. After the extension, they may represent Lifshitz black holes [28-49]. The distinguishable features of these solutions are that: (i) they are exact vacuum solutions of the HL gravity without any matter; and (ii) the corresponding metrics are given explicitly and in closed forms, in contrast to the relativistic cases in which most of the solutions were constructed numerically [28-52]. We expect that this will facilitate considerably the studies of the holographic dual between the non-relativistic Lifshitz QFTs and theories of quantum gravity.

It should be noted that the definition of black holes in the HL gravity is subtle [61-71], because of the inclusions of high-order derivative operators, for which the dispersion relationship is in general becomes nonlinear,

$$
E^{2}=c_{p}^{2} p^{2}\left(1+\alpha_{1}\left(\frac{p}{M_{*}}\right)^{2}+\alpha_{2}\left(\frac{p}{M_{*}}\right)^{4}\right)
$$

where $E$ and $p$ denote, respectively, the energy and momentum of the particle, and $c_{p}$ and $\alpha_{i}$ are coefficients, depending on the particular specie of the particle, while $M_{*}$ denotes the suppression energy scale of the higher-dimensional operators. Then, both of the phase and group velocities of the particle become unbounded as its momentum increases. As a result, black holes may not exist at all in the HL theory [62-71]. However, in the IR the high-order terms of $p$ are negligible, and the first term in eq. (1.3) becomes dominant, so one may still define black holes, following what was done in GR [72-80]. Therefore, in this paper we shall consider the HL gravity in the IR limit. Nevertheless, cautions must be taken even in this limit: because of the Lorentz violation of the theory, spin- 0 gravitons generically appear [54-57], whose velocity in general is different from that of light. To avoid the Cherenkov effects, it is necessary to require it to be no smaller than the speed of light $[81,82]$. As a result, even they are initially trapped inside the horizons, the spin-0 gravitons can escape from them and make the definition of black holes given in GR invalid. ${ }^{2}$ Fortunately, it was shown recently that universal horizons might exist inside the event horizons of GR, where the preferred time foliation simply ceases to penetrate them

\footnotetext{
${ }^{2}$ One might argue that black holes then can be defined in terms of the light cone of these spin-0 gravi-
} 
within any finite time [85]. Universal horizons have already attracted lot of attention, and various interesting results have been obtained [86-90]. For more detail regarding to black holes in the HL gravity, we refer readers to [61-71, 85-90], and references therein.

To simplify the technique issues and be comparable to the studies carried out in [60], in this paper we shall restrict ourselves only to $(2+1)$ dimensional spacetimes, ${ }^{3}$ although we find that exact vacuum solutions of the HL gravity in any dimensional spacetimes exist, and have similar space-time structures [91]. Specifically, the paper is organized as follows: in section 2, we give a brief introduction to the non-projectable HL theory in $(2+1)$ dimensions. In section 3 , we first present all the static diagonal vacuum solutions of the HL theory, and then study their local and global structures. We find that the Lifshitz spacetime (1.1) is only one of the whole class of solutions, and the rest of them can represent either Lifshitz solitons, in which space-time is not singular, or generalized BTZ black holes. Some solutions represent incomplete space-time, and extensions beyond certain horizons are needed. After the extension, they may represent Lifshitz black holes [28-49]. In section 4 , we construct several classes of static non-diagonal $\left(g_{t r} \neq 0\right)$ vacuum solutions of the HL theory, and find that there exist similar space-time structures as found in the diagonal case. In section 5 , we present our main conclusions.

\section{Non-projectable HL gravity}

Because of the anisotropic scaling, the symmetry of general covariance is necessarily broken. Hořava assumed that it is broken only down to the foliation-preserving diffeomorphism,

$$
\delta t=-f(t), \quad \delta x^{i}=-\zeta^{i}(t, \mathbf{x}),
$$

often denoted by $\operatorname{Diff}(M, \mathcal{F})$. Then, the lapse function $N$, shift vector $N^{i}$, and 3-spatial metric $g_{i j}$ in the Arnowitt-Deser-Misner (ADM) decompositions [92, 93] transform as

$$
\begin{aligned}
\delta N & =\zeta^{k} \nabla_{k} N+\dot{N} f+N \dot{f}, \\
\delta N_{i} & =N_{k} \nabla_{i} \zeta^{k}+\zeta^{k} \nabla_{k} N_{i}+g_{i k} \dot{\zeta}^{k}+\dot{N}_{i} f+N_{i} \dot{f}, \\
\delta g_{i j} & =\nabla_{i} \zeta_{j}+\nabla_{j} \zeta_{i}+f \dot{g}_{i j},
\end{aligned}
$$

where $\dot{f} \equiv d f / d t, \nabla_{i}$ denotes the covariant derivative with respect to $g_{i j}, N_{i}=g_{i k} N^{k}$, and $\delta g_{i j} \equiv \tilde{g}_{i j}\left(t, x^{k}\right)-g_{i j}\left(t, x^{k}\right)$, etc.

In the HL gravity, the development usually follows two different lines [54-57], one is with the projectability condition, in which the lapse function is a function of $t$ only, and the other is without the projectability condition, in which the lapse function is a function of both time and space coordinates, that is,

$$
N=N(t, x) .
$$

In this paper, we shall assume this non-projectable condition.

tons. However, due to the Lorentz violation, other excitations with different speeds might exist, unless a mechanism is invented to prevent this from happening, for example, by assuming that the matter sector satisfies the Lorentz symmetry up to the Planck scale [83, 84].

${ }^{3}$ In (2+1)-dimensions, observational constraints from the Cherenkov effects are out of question, so in principle the speed of the spin-0 gravitons can be smaller than that of light. 
In (2+1)-dimensional spacetimes, the Riemann and Ricci tensors $R_{i j k l}$ and $R_{i j}$ of the 2-dimensional spatial surfaces $t=$ constant are uniquely determined by the 2 -dimensional Ricci scalar $R$ via the relations [94],

$$
\begin{aligned}
R_{i j k l} & =\frac{1}{2}\left(g_{i k} g_{j l}-g_{i l} g_{j k}\right) R, \\
R_{i j} & =\frac{1}{2} g_{i j} R,(i, j=1,2) .
\end{aligned}
$$

Then, the general action of the HL theory without the projectability condition in $(2+1)$ dimensional spacetimes can be cast in the form,

$$
S=\zeta^{2} \int d t d^{2} x N \sqrt{g}\left(\mathcal{L}_{K}-\mathcal{L}_{V}+\zeta^{-2} \mathcal{L}_{M}\right)
$$

where $g=\operatorname{det}\left(g_{i j}\right), \zeta^{2}=1 /(16 \pi G)$, and

$$
\begin{aligned}
\mathcal{L}_{K} & =K_{i j} K^{i j}-\lambda K^{2}, \\
K_{i j} & =\frac{1}{2 N}\left(-\dot{g}_{i j}+\nabla_{i} N_{j}+\nabla_{j} N_{i}\right),
\end{aligned}
$$

where $\lambda$ is a dimensionless coupling constant. $\mathcal{L}_{M}$ is the Lagrangian of matter fields.

The potential $\mathcal{L}_{V}$ can be easily obtained from [95-97], by noting the special relations (2.4) in (2+1)-dimensions and the fact that to keep the theory power-counting renormalizable only up to the fourth-order derivative terms are needed. Then, it can be cast in the form [95-97],

$$
\begin{aligned}
\mathcal{L}_{V}= & \gamma_{0} \zeta^{2}+\beta a_{i} a^{i}+\gamma_{1} R+\frac{\gamma_{2}}{\zeta^{2}} R^{2} \\
& +\frac{1}{\zeta^{2}}\left[\beta_{1}\left(a_{i} a^{i}\right)^{2}+\beta_{2}\left(a_{i}^{i}\right)^{2}+\beta_{3}\left(a_{i} a^{i}\right) a_{j}^{j}+\beta_{4} a^{i j} a_{i j}+\beta_{5}\left(a_{i} a^{i}\right) R+\beta_{6} R a^{i}{ }_{i}\right]
\end{aligned}
$$

where $\beta\left(\equiv-\beta_{0}\right), \beta_{n}$ and $\gamma_{n}$ are all dimensionless and arbitrary coupling constants, and

$$
a_{i} \equiv \frac{N_{, i}}{N}, \quad a_{i j} \equiv \nabla_{i} a_{j}
$$

\subsection{Field equations}

Variation of the action (2.5) with respect to the lapse function $N$ yields the Hamiltonian constraint

$$
\mathcal{L}_{K}+\mathcal{L}_{V}^{R}+F_{V}=8 \pi G J^{t}
$$

where

$$
\begin{aligned}
J^{t} & =2 \frac{\delta\left(N \mathcal{L}_{M}\right)}{\delta N}, \\
\mathcal{L}_{V}^{R} & =\gamma_{0} \zeta^{2}+\gamma_{1} R+\frac{\gamma_{2}}{\zeta^{2}} R^{2},
\end{aligned}
$$

and $F_{V}$ is given by eq. (A.1) in appendix A. 
Variation with respect to the shift vector $N_{i}$ yields the momentum constraint

$$
\nabla_{j} \pi^{i j}=8 \pi G J^{i}
$$

where

$$
\pi^{i j} \equiv-K^{i j}+\lambda K g^{i j}, \quad J^{i} \equiv-\frac{\delta\left(N \mathcal{L}_{M}\right)}{\delta N_{i}} .
$$

The dynamical equations are obtained by varying $S$ with respect to $g_{i j}$, and are given by

$$
\begin{aligned}
\frac{1}{\sqrt{g} N} & \frac{\partial}{\partial t}\left(\sqrt{g} \pi^{i j}\right)+2\left(K^{i k} K_{k}^{j}-\lambda K K^{i j}\right) \\
& -\frac{1}{2} g_{i j} \mathcal{L}_{K}+\frac{1}{N} \nabla_{k}\left(\pi^{i k} N^{j}+\pi^{k j} N^{i}-\pi^{i j} N^{k}\right)-F^{i j}-F_{a}^{i j}=8 \pi G \tau^{i j},
\end{aligned}
$$

where

$$
\begin{aligned}
F^{i j} & \equiv \frac{1}{\sqrt{g} N} \frac{\delta\left(-\sqrt{g} N \mathcal{L}_{V}^{R}\right)}{\delta g_{i j}}=\sum_{s=0}^{s=2} \hat{\gamma}_{s} \zeta^{n_{s}}\left(F_{s}\right)^{i j} \\
F_{a}^{i j} & \equiv \frac{1}{\sqrt{g} N} \frac{\delta\left(-\sqrt{g} N \mathcal{L}_{V}^{a}\right)}{\delta g_{i j}}=\sum_{s=0}^{s=6} \hat{\beta}_{s} \zeta^{m_{s}}\left(F_{s}^{a}\right)^{i j} \\
\tau^{i j} & \equiv \frac{2}{\sqrt{g} N} \frac{\delta\left(\sqrt{g} N \mathcal{L}_{M}\right)}{\delta g_{i j}}
\end{aligned}
$$

with

$$
\begin{aligned}
\hat{\gamma}_{s} & =\left(\gamma_{0}, \gamma_{1}, \gamma_{2}\right), \\
n_{s} & =(2,0,-2), \\
\hat{\beta}_{s} & =\left(\beta, \beta_{n}\right) \quad(n=1,2, \ldots, 6), \\
m_{s} & =(0,-2,-2,-2,-2,-2,-2) .
\end{aligned}
$$

The functions $\left(F_{s}\right)^{i j}$ and $\left(F_{s}^{a}\right)^{i j}$ are given by eq. (A.2) in appendix A.

In addition, the matter components $\left(J^{t}, J^{i}, \tau^{i j}\right)$ satisfy the conservation laws of energy and momentum,

$$
\begin{array}{r}
\int d^{3} x \sqrt{g} N\left[\dot{g}_{i j} \tau^{i j}-\frac{1}{\sqrt{g}} \partial_{t}\left(\sqrt{g} J^{t}\right)+\frac{2 N_{i}}{\sqrt{g} N} \partial_{t}\left(\sqrt{g} J^{i}\right)\right]=0, \\
\frac{1}{N} \nabla^{i}\left(N \tau_{i k}\right)-\frac{1}{\sqrt{g} N} \partial_{t}\left(\sqrt{g} J_{k}\right)-\frac{J^{t}}{2 N} \nabla_{k} N-\frac{N_{k}}{N} \nabla_{i} J^{i}-\frac{J^{i}}{N}\left(\nabla_{i} N_{k}-\nabla_{k} N_{i}\right)=0 .
\end{array}
$$

\subsection{Ghost-free and stability conditions}

When $\Lambda=0$, the flat space-time,

$$
\left(N, N_{i}, g_{i j}\right)=\left(1,0, \delta_{i j}\right),
$$

is a solution of the above HL theory in the IR. It can be shown that in this model spin-0 gravitons appear due to the reduced symmetry (2.1) [60], in contrast to GR. The speed of these particles is given by,

$$
c_{s}^{2}=-\frac{\gamma_{1}^{2}(1-\lambda)}{\beta(1-2 \lambda)} .
$$


The ghost-free and stability of the flat background require [60],

$$
\begin{aligned}
\frac{1-\lambda}{1-2 \lambda} & >0, \\
-\frac{1-\lambda}{\beta(1-2 \lambda)} & \geq 0,
\end{aligned}
$$

which yield

$$
\begin{gathered}
\beta<0 \\
\text { (i) } \lambda \geq 1, \quad \text { or } \quad(i i) \lambda \leq \frac{1}{2} .
\end{gathered}
$$

\section{Static vacuum solutions in the IR limit}

The general static spacetimes without the projectability condition are described by,

$$
\begin{aligned}
N & =r^{z} f(r), \quad N^{i}=\left(N^{r}(r), 0\right), \\
g_{i j} d x^{i} d x^{j} & =\frac{g^{2}(r)}{r^{2}} d r^{2}+r^{2} d x^{2},
\end{aligned}
$$

in the coordinates $(t, r, x)$. Then, we find that

$$
\begin{aligned}
K_{i j} & =\frac{g}{r^{z+1} f}\left(\left(\frac{H}{r}\right)^{\prime} \delta_{i}^{r} \delta_{i}^{r}+\frac{r^{2}}{g^{2}} H \delta_{i}^{x} \delta_{i}^{x}\right), \\
R_{i j} & =\frac{r g^{\prime}-g}{r^{2} g} \delta_{i}^{r} \delta_{j}^{r}+\frac{r^{2}\left(r g^{\prime}-g\right)}{g^{3}} \delta_{i}^{x} \delta_{j}^{x}, \\
a_{i} & =\frac{\left(z f+r f^{\prime}\right)}{r f} \delta_{i}^{r}, \quad H \equiv g N^{r},
\end{aligned}
$$

where a prime denotes the ordinary derivative with respect to $r$.

In the IR, all the high-order derivative operators proportional to the coupling constants $\gamma_{2,3,4}$ and $\beta_{1, \ldots, 4}$ are suppressed by $1 / M_{*}^{n-2}$, so are negligible for $E \ll M_{*}$, where $n$ denotes the order of the operator, and $M_{*}[=\sqrt{1 /(8 \pi G)}]$ is the Planck mass of the HL theory (which can be different from that of GR). Therefore, in the IR these high-order terms can be safely set to zero. Then, for the vacuum solutions where

$$
\tau^{i j}=J^{t}=J^{i}=0
$$

the Hamiltonian and momentum constraints (2.9) and (2.12) reduce, respectively, to

$$
\begin{aligned}
\frac{1}{2 r^{2 z} f^{2}}\left[(1-\lambda)\left(H^{\prime}\right)^{2}-2 H\left(\frac{H}{r}\right)^{\prime}\right]+\Lambda g^{2} \\
-\beta\left[g\left(\frac{r W}{g}\right)^{\prime}+\frac{W^{2}}{2}\right]+\gamma_{1}\left(r \frac{g^{\prime}}{g}-1\right)=0, \\
\left(\frac{1}{r^{z-1} f g}\right)^{\prime} H+(\lambda-1) r^{2}\left(\frac{H^{\prime}}{r^{z} f g}\right)^{\prime}=0,
\end{aligned}
$$


where

$$
W \equiv z+\frac{r f^{\prime}}{f}, \quad \Lambda \equiv \frac{\gamma_{0} \zeta^{2}}{2} .
$$

The $(r r)$ and $(x x)$ components of the dynamical equations (2.14) are

$$
\begin{aligned}
&(1-\lambda) g\left[\frac{\left(H^{\prime}\right)^{2}}{2 r^{z} g f}-H\left(\frac{H^{\prime}}{r^{z} g f}\right)^{\prime}\right]-\frac{H\left(r^{z-2} g f H\right)^{\prime}}{r^{2 z-1} g f^{2}} \\
&+r^{z} f\left[\Lambda g^{2}-\gamma_{1} W-\frac{\beta}{2} W^{2}\right]=0, \\
&(1-\lambda) g\left[\frac{\left(H^{\prime}\right)^{2}}{2 r^{z} g f}+H\left(\frac{H^{\prime}}{r^{z} g f}\right)^{\prime}\right]-g r\left[\frac{H}{r^{z} g f}\left(\frac{H}{r}\right)^{\prime}\right]^{\prime} \\
&+r^{z} f\left\{\Lambda g^{2}-\gamma_{1}\left[W^{2}-r g\left(\frac{W}{g}\right)^{\prime}\right]+\frac{\beta}{2} W^{2}\right\}=0 .
\end{aligned}
$$

It can be shown that eq. (3.7) is not independent, and can be obtained from eqs. (3.3)-(3.6). Thus, we need only consider eqs. (3.3), (3.4) and (3.6) for the three unknowns, $f(r), g(r)$ and $N^{r}(r)$.

In the rest of this section, we consider only the diagonal case where $N^{r}=0$, and leave the studies of the non-diagonal case $N^{r} \neq 0$ to the next section.

When $N^{r}=0$ (or $H=0$ ), it is clear that eq. (3.4) is trivially satisfied, while eqs. (3.3) and (3.6) reduce to

$$
\begin{aligned}
\Lambda g^{2}-\beta\left[g\left(\frac{r W}{g}\right)^{\prime}+\frac{W^{2}}{2}\right]-\gamma_{1} g\left(\frac{r}{g}\right)^{\prime} & =0, \\
\Lambda g^{2}-\gamma_{1} W-\frac{\beta}{2} W^{2} & =0 .
\end{aligned}
$$

From eq. (3.9), we obtain

$$
W_{ \pm}=\frac{s \pm s r_{*}(r)}{1-s}
$$

where

$$
s \equiv \frac{\gamma_{1}}{\gamma_{1}-\beta}, \quad r_{*}(r) \equiv \sqrt{1+\frac{2 \beta \Lambda}{\gamma_{1}^{2}} g(r)^{2}} .
$$

Inserting the above into eq. (3.8), we obtain a master equation for $r_{*}(r)$,

$$
(s-1) r r_{*}^{\prime}+\left(r_{*}^{2}-1\right)\left(r_{*} \pm s\right)=0 .
$$

To solve this equation, let us consider the cases with different $s$, separately.

\subsection{Lifshitz spacetime}

A particular solution of eq. (3.12) is $r_{*}=\mp s$. Then, from eqs. (3.5) and (3.10), we find that

$$
f=f_{0}, \quad z=s=\frac{\gamma_{1}}{\gamma_{1}-\beta},
$$


while eq. (3.11) yields,

$$
g=g_{0}, \quad \Lambda=\frac{\gamma_{1}^{2}\left(2 \gamma_{1}-\beta\right)}{2 g_{0}^{2}\left(\gamma_{1}-\beta\right)^{2}}
$$

where $f_{0}$ and $g_{0}$ are two constants. Thus, the corresponding line element takes the form,

$$
d s^{2}=L^{2}\left\{-\left(\frac{r}{\ell}\right)^{2 z} d t^{2}+\left(\frac{\ell}{r}\right)^{2} d r^{2}+\left(\frac{r}{\ell}\right)^{2} d x^{2}\right\},
$$

where $f_{0} \equiv L / \ell^{z}$ and $g_{0} \equiv L \ell$. Rescaling the coordinates $t, r, x$, without loss of the generality, one can always set $L=\ell=1$. The above solution is exactly the one obtained in [60] for the case $D=1$. The metric is invariant under the anisotropic scalings,

$$
t \rightarrow b^{-z} t, \quad r \rightarrow b r, \quad x \rightarrow b^{-1} x
$$

In addition, from eq. (3.2) we find that the corresponding curvature $R$ is given by

$$
R=-\frac{4 \Lambda\left(\gamma_{1}-\beta\right)^{2}}{\gamma_{1}^{2}\left(2 \gamma_{1}-\beta\right)}
$$

which is a constant. However, it can be shown that the space-time at $r=0$ is singular, and the nature of it is null [21, 22].

\subsection{Asymptotical Lifshitz spacetimes}

In order for a static solution to be asymptotically to the Lifshitz solution (3.15), the functions $f$ and $g$ must be

$$
\lim _{r \rightarrow \infty} f(r)=\lim _{r \rightarrow \infty} g^{-1}(r)=1 .
$$

It is remarkable to note that eqs. (3.10) and (3.12) indeed allow such solutions,

$$
\begin{aligned}
\frac{W}{r} & \simeq \frac{f^{\prime}}{f} \simeq 0, \\
r_{*}(r) & \simeq r_{*}^{0},
\end{aligned}
$$

for $r \gg 1$, where $r_{*}^{0}$ is a constant, and the asymptotical conditions (3.18) require

$$
r_{*}^{0}=\sqrt{1+\frac{2 \beta \Lambda}{\gamma_{1}^{2}}} .
$$

To solve eq. (3.12), let us first write it in the form,

$$
\frac{d r}{r}=\left(\frac{1 \pm s}{r_{*}+1}+\frac{1 \mp s}{r_{*}-1}-\frac{2}{r_{*} \pm s}\right) \frac{d r_{*}}{2(1+s)}
$$

which has the general solutions,

$$
r_{ \pm}\left(r_{*}\right)=r_{H}\left|r_{*}+1\right|^{\frac{1 \pm s}{2(1+s)}}\left|r_{*}-1\right|^{\frac{1 \mp s}{2(1+s)}}\left|r_{*} \pm s\right|^{-\frac{1}{s+1}}
$$


where $r_{H}$ is an integration constant, and $r_{+}\left(r_{-}\right)$corresponds to the choice $W=W_{+}$ $\left(W=W_{-}\right)$. It is interesting to note that we can obtain $r_{+}\left(r_{*}\right)$ from $r_{-}\left(r_{*}\right)$ by replacing $r_{*}$ by $-r_{*}$, i.e., $r_{+}\left(r_{*}\right)=r_{-}\left(-r_{*}\right)$. The same are true for $W_{ \pm}$, and the functions $f\left(r_{*}\right)$ and $g\left(r_{*}\right)$ to be derived below. Therefore, in the following we shall take the region $r_{*}<0$ as a natural extension of the one defined by eq. (3.11), and, without loss of the generality, in the following we shall consider only the solution $r_{+}\left(r_{*}\right)$. Then, from eq. (3.11) we find that

$$
g^{2}(r)=\frac{\gamma_{1}^{2}}{2 \beta \Lambda}\left(r_{*}^{2}-1\right),
$$

while from eqs. (3.5) and (3.10), we find that

$$
\frac{d f(r)}{f(r)}=\frac{s-z(1-s)+s r_{*}}{(1-s)} \frac{d r}{r} .
$$

Inserting eq. (3.21) with the upper signs into the above expression and then integrating it, we find

$$
f(r)=f_{0}\left|r_{*}+1\right|^{-\frac{z}{2}}\left|r_{*}-1\right|^{\frac{2 s-z(1-s)}{2(1+s)}}\left|r_{*}+s\right|^{\frac{z-s}{1+s}},
$$

where $f_{0}$ is an integration constant. In summary, we obtain the following general solutions,

$$
\begin{aligned}
r^{2 z} f^{2}(r) & =N_{0}^{2}\left|\frac{r_{*}-1}{r_{*}+s}\right|^{\frac{2 s}{1+s}}, \\
g^{2}(r) & =\frac{\gamma_{1}^{2}}{2 \beta \Lambda}\left(r_{*}^{2}-1\right),
\end{aligned}
$$

where $N_{0} \equiv f_{0} r_{H}^{z}$. Then, in terms of $r_{*}$ the line element becomes

$$
\begin{aligned}
d s^{2}=-N_{0}^{2}\left|\frac{r_{*}-1}{r_{*}+s}\right|^{\frac{2 s}{1+s}} d t^{2}+\frac{\gamma_{1}^{2}(1-s)^{2} d r_{*}^{2}}{2 \beta \Lambda\left(r_{*}^{2}-1\right)\left(r_{*}+s\right)^{2}} & \\
& +r_{H}^{2}\left|\frac{r_{*}-1}{r_{*}+s}\right|^{\frac{1-s}{1+s}}\left|\frac{r_{*}+1}{r_{*}+s}\right| d^{2} x .
\end{aligned}
$$

As noted previously, the functions $g\left(r_{*}\right), f\left(r_{*}\right)$, and the metric given in the present case are well-defined for $r_{*}<0$. So, in the following we simply generalize the above solutions to $r_{*} \in(-\infty,+\infty)$. Then, from eq. (3.2) we find that

$$
R=\frac{4 \beta \Lambda\left(r_{*}+s-1\right)}{\gamma_{1}^{2}(1-s)\left(r_{*}-1\right)} .
$$

Thus, the space-time is always singular at $r_{*}=+1$, unless $s=1$ that will be considered in the next subsection. Actually, near $r_{*} \simeq 1$, we have

$$
r \simeq L_{0}\left|r_{*}-1\right|^{\frac{1-s}{2(1+s)}},
$$

where $L_{0} \equiv \sqrt{2} r_{H}|1+s|^{-1 /(1+s)}$, and the metric (3.27) becomes

$$
d s^{2} \simeq\left(\frac{r}{L_{0}}\right)^{\frac{4 s}{1-s}}\left[-\tilde{L}_{0}^{2} d t^{2}+\left(\frac{\epsilon^{+} \gamma_{1}^{2}}{\beta \Lambda L_{0}^{2}}\right) d r^{2}\right]+r^{2} d x^{2},\left(r_{*} \simeq 1\right),
$$


where $\tilde{L}_{0}=|1+s|^{-s /(1+s)} N_{0}$ and $\epsilon^{+} \equiv \operatorname{sign}\left(r_{*}-1\right)$. Recall that the stability and ghost-free conditions require $\beta<0$, as given by eq. (2.23). Then, for the metric to have a proper signature in the neighborhood $r_{*}=1$, we must assume that

$$
\epsilon^{+} \Lambda<0
$$

Note that the metric is also singular at $r_{*}=-1$. However, this singularity is not a scalar one, as shown above. In fact, when $r_{*} \simeq-1$, we have

$$
r \simeq \tilde{r}_{0}\left|r_{*}+1\right|^{1 / 2}
$$

where $\tilde{r}_{0} \equiv 2^{(1-s) /[2(1+s)]} r_{H}|1-s|^{-1 /(1+s)}$. Then, the metric (3.27) takes the asymptotical form,

$$
d s^{2} \simeq-\tilde{N}_{0}^{2} d t^{2}+\left(\frac{\epsilon^{-} \gamma_{1}^{2}}{-\beta \Lambda \tilde{r}_{0}^{2}}\right) d r^{2}+r^{2} d^{2} x,\left(r_{*} \simeq-1\right),
$$

which is locally flat, where $\tilde{N}_{0} \equiv N_{0}|2 /(1-s)|^{s /(1+s)}$ and $\epsilon^{-} \equiv \operatorname{sign}\left(r_{*}+1\right)$. Since $\beta<0$, the cosmological constant $\Lambda$ needs to be chosen so that

$$
\epsilon^{-} \Lambda>0
$$

in order for the metric to have a proper signature in the neighborhood of $r_{*}=-1$. To study further the solutions in the neighborhood of $r_{*}=-1$, let us calculate the tidal forces. Following $[21,22]$, we can show that the radial timelike geodesics are given by

$$
\frac{d r_{*}}{d \tau}= \pm \xi E\left|r_{*}+1\right|^{\frac{1}{2}}\left|r_{*}-1\right|^{\frac{1-s}{2(1+s)}}\left|r_{*}+s\right|^{\frac{1+2 s}{1+s}} \sqrt{1-\frac{N_{0}^{2}}{E^{2}}\left|\frac{r_{*}-1}{r_{*}+s}\right|^{\frac{2 s}{1+s}}},
$$

where $E$ is an integration constant, and $\tau$ is the proper time. The constant $\xi$ is defined by

$$
\xi \equiv \sqrt{\frac{2 \beta \Lambda \epsilon^{+} \epsilon^{-}}{\gamma_{1}^{2}(1-s)^{2} N_{0}^{2}}} .
$$

The "+" and "-" denote, respectively, the outgoing and ingoing radial geodesics. In what follows we would like to calculate the tidal forces felt by the freely falling explorer at $r_{*}=-1$. We therefore choose the following orthonormal frame

$$
\begin{aligned}
& e_{(0)}^{\mu}=\left(\frac{E}{N_{0}^{2}}\left|\frac{r_{*}+s}{r_{*}-1}\right|^{\frac{2 s}{1+s}},-\left|\frac{d r_{*}}{d \tau}\right|, 0\right),
\end{aligned}
$$

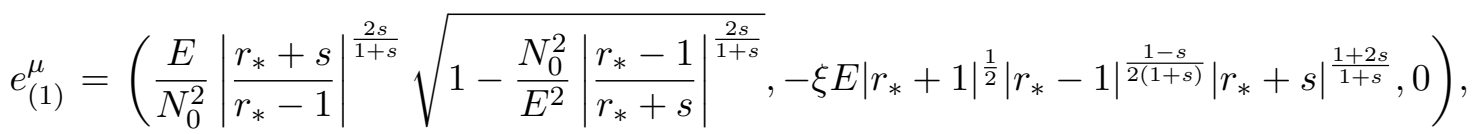

$$
\begin{aligned}
& e_{(2)}^{\mu}=\left|\frac{r_{*}+s}{r_{*}-1}\right|^{\frac{1-s}{2(1+s)}}\left|\frac{r_{*}+s}{r_{*}+1}\right|^{\frac{1}{2)}}\left(0,0, \frac{1}{r_{H}}\right) \text {, }
\end{aligned}
$$

which are obviously orthonormal

$$
g_{\mu \nu} e_{(a)}^{\mu} e_{(b)}^{\nu}=\eta_{a b},
$$


with $\eta_{a b}$ being the Minkowski metric. The tidal forces are measured by the components of the Riemann curvature tensor with respect to the above orthonormal frame, i.e.,

$$
R_{a b c d}=R_{\mu \nu \rho \sigma} e_{(a)}^{\mu} e_{(b)}^{\nu} e_{(c)}^{\rho} e_{(d)}^{\sigma} .
$$

One can show that in the limit $r_{*} \rightarrow-1$, the nonzero components of $R_{a b c d}$ are given by

$$
\begin{aligned}
& R_{0101} \simeq \frac{\epsilon^{-}}{2} \xi^{2} N_{0}^{2} s(1-s) \\
& R_{0202} \simeq \frac{\epsilon^{-}}{2} \xi^{2} N_{0}^{2}(s-1)(s-2)-4 \xi^{2} E^{2}\left|\frac{s-1}{2}\right|^{\frac{2+4 s}{1+s}}, \\
& R_{1212} \simeq \frac{\epsilon^{-}}{2} \xi^{2} N_{0}^{2} s(s-1)-4 \xi^{2} E^{2}\left|\frac{s-1}{2}\right|^{\frac{2+4 s}{1+s}}, \\
& R_{0212} \simeq-\epsilon^{-} \xi^{2} E^{2} 2^{\frac{-2 s}{1+s}}|s-1|^{\frac{2+3 s}{1+s}} \sqrt{E^{2}|s-1|^{\frac{2 s}{1+s}}-2^{\frac{2 s}{1+s}} N_{0}^{2}} .
\end{aligned}
$$

Clearly, they are all finite and there is no singularity at $r_{*}=-1$ ( or $r=0$ ), even the null curvature ones, as found in the Lifshitz space-time at the origin $r=0$ [21, 22].

On the other hand, as $r_{*} \rightarrow-s$, we have

$$
r \rightarrow \hat{r}_{0}\left|r_{*}+s\right|^{-\frac{1}{1+s}}
$$

where $\hat{r}_{0} \equiv r_{H}|s-1|^{1 / 2}|s+1|^{(1-s) /[2(s+1)]}$. Then, the metric (3.27) takes the asymptotical form,

$$
d s^{2} \simeq-r^{2 s} d \hat{t}^{2}+\frac{d r^{2}}{r^{2}}+r^{2} d^{2} x,\left(r_{*} \rightarrow-s\right),
$$

which is precisely the Lifshitz space-time (3.15) with $z=s$, where $\hat{t}=N_{0} r_{H}^{-s} \mid(1+s) /(1-$ $s)\left.\right|^{-s / 2} t$. Note that in writing the above metric we had used a generalized condition (3.20) for $r_{*}^{0}=-s$, so that

$$
\gamma_{1}^{2}\left(s^{2}-1\right)=2 \beta \Lambda
$$

The behavior $r$ vs $r_{*}$ depends on the values of $s$. Therefore, in the following let us consider the cases with different values of $s$, separately.

\subsection{1 $s>1$}

In this case, we have

$$
r\left(r_{*}\right)= \begin{cases}r_{H}, & r_{*} \rightarrow-\infty \\ \infty, & r_{*}=-s \\ 0, & r_{*}=-1 \\ \infty, & r_{*}=+1 \\ r_{H}, & r_{*} \rightarrow+\infty\end{cases}
$$

figure 1 shows the function $r\left(r_{*}\right)$ vs $r_{*}$, from which we can see that the region $r \in[0, \infty)$ is mapped into the region $r_{*} \in[-1,+1)$ or $r_{*} \in(-s,-1]$. The region $r_{*} \in(-\infty,-s)$ or $r_{*} \in(+1,+\infty)$ is mapped into the one $r \in\left(r_{H},+\infty\right)$. 


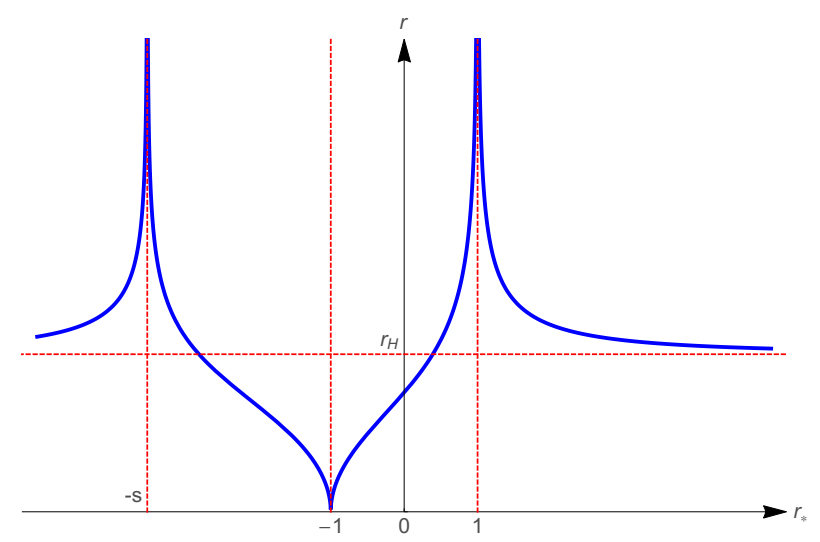

Figure 1. The function $r \equiv r_{+}\left(r_{*}\right)$ defined by eq. (3.22) vs $r_{*}$ for $s>1$. The space-time is singular at $r_{*}=1$, locally flat at $r_{*}=-1$ and asymptotically approaching the Lifshitz space-time (3.15) with $z=s$ as $r_{*} \rightarrow-s$.

Considering the fact that the space-time is singular at $r_{*}=1$, a physically well-defined region is $r_{*} \in(-s,-1]$, which corresponds to the region $r \in[0,+\infty)$ ). At $r=0$ (or $r_{*}=-1$ ), the space-time is locally flat, and as $r \rightarrow \infty$ (or $r_{*} \rightarrow-s$ ), it is asymptotically approaching to the Lifshitz space-time (3.15) with $z=s$. Therefore, in this region the solution represents a Lifshitz soliton [50-52]. Since $s>1$, then in the region $r_{*} \in(-s,-1]$, we have $\epsilon^{-}=\left.\operatorname{sign}\left(r_{*}+1\right)\right|_{r_{*} \simeq-1}=-1$. Thus, the conditions (3.34) and (3.43) require

$$
\Lambda<0,(s>1) \text {. }
$$

To study the solutions further, let us rewrite eq. (3.22) (with $r=r_{+}$) in the form

$$
\left(\frac{r}{r_{H}}\right)^{2}=\frac{(s-1) \epsilon^{-}}{s+1}\left(\epsilon^{+} \mathfrak{R}^{\frac{2}{1-s}}+\frac{2 \epsilon^{s}}{s-1} \mathfrak{R}\right),
$$

where $\epsilon^{s} \equiv \operatorname{sign}\left(r_{*}+s\right)$ and

$$
\mathfrak{R} \equiv\left|\frac{r_{*}-1}{r_{*}+s}\right|^{\frac{1-s}{1+s}} .
$$

It should be noted that the above two equations are valid not only for $s>1$, but also for other values of $s$.

In general it is difficult to obtain an explicit expression of $\mathfrak{R}$ for any given $s$ in terms of $r$. Therefore, in the following let us consider the representative case $s=3$, for which eqs. (3.46) and (3.47) reduce to,

$$
\begin{aligned}
\left(\frac{r}{r_{H}}\right)^{2} & =\frac{\epsilon^{-}}{2 \mathfrak{R}}\left(\epsilon^{+}+\epsilon^{s} \mathfrak{R}^{2}\right), \\
\mathfrak{R} & =\left|\frac{r_{*}+3}{r_{*}-1}\right|^{1 / 2} .
\end{aligned}
$$

As shown in figure 1 , the whole axis $r_{*} \in(-\infty, \infty)$ is divided into four different segments, and in each of them the space-time has different properties. Therefore, in the following we consider the space-time in each of the four segments, separately. 
(a) In the region $r_{*} \in(-\infty,-3]$, we have $\epsilon^{+}=\epsilon^{-}=\epsilon^{s}=-1$. Then, from eq. (3.48) we obtain

$$
\begin{aligned}
& \Re=\left(\frac{r}{r_{H}}\right)^{2}\left(1 \pm \sqrt{1-\left(\frac{r_{H}}{r}\right)^{4}}\right), \\
& r_{*}=\frac{\mathfrak{R}^{2}+3}{\mathfrak{R}^{2}-1} .
\end{aligned}
$$

Since $\mathfrak{R} \in[0,1)$, as it can be seen from eq. (3.48), we find that only the root $\mathfrak{R}_{-}$ satisfies this condition. On the other hand, from eq. (3.26) we find,

$$
r^{2 z} f^{2}=\frac{N_{0}^{2}}{\mathfrak{R}_{-}^{3}}, \quad g^{2}=\left(\frac{4 \gamma_{1}^{2}}{\beta \Lambda}\right) \frac{1+\mathfrak{R}_{-}^{2}}{\left(\mathfrak{R}_{-}^{2}-1\right)^{2}},
$$

where

$$
\mathfrak{R}_{-}=\frac{\left(\frac{r_{H}}{r}\right)^{2}}{1+\sqrt{1-\left(\frac{r_{H}}{r}\right)^{4}}}= \begin{cases}1, & r=r_{H} \\ 0, & r=\infty\end{cases}
$$

Thus, we obtain the following asymptotic behavior

$$
f^{2} \rightarrow 8 f_{0}^{2}, \quad g^{2} \rightarrow \frac{6 \gamma_{1}}{\Lambda},
$$

which is just what is expected. In terms of $r$, the metric can be written in the form,

$$
d s^{2}=-\frac{r^{6}}{8}\left(1+\sqrt{1-\left(\frac{r_{H}}{r}\right)^{4}}\right)^{3} d t^{2}+\frac{1+\sqrt{1-\left(\frac{r_{H}}{r}\right)^{4}}}{2\left(1-\left(\frac{r_{H}}{r}\right)^{4}\right)} \frac{r^{2}}{r^{2}}+r^{2} d x^{2} .
$$

Note that in writing the above metric, we had used the asymptotic condition (3.13) and (3.14), and meanwhile rescaled $t$ by $t \rightarrow 2 \sqrt{2} f_{0} t$. From the above expressions it can be seen clearly that the solution is valid only in the region $r \geq r_{H}$, and $r=r_{H}$ represents a horizon. To have a complete space-time, extension beyond this surface is needed.

(b) In the region $r_{*} \in(-3,-1]$, we have $\epsilon^{+}=\epsilon^{-}=-\epsilon^{s}=-1$. Then, we find that

$$
\begin{aligned}
\mathfrak{R} & =\left(\frac{r}{r_{H}}\right)^{2}\left(\sqrt{1+\left(\frac{r_{H}}{r}\right)^{4}}-1\right), \\
r_{*} & =\frac{\mathfrak{R}^{2}-3}{\mathfrak{R}^{2}+1},
\end{aligned}
$$

are solutions to eq. (3.46). This immdeiately leads to the line element,

$$
d s^{2}=-\frac{r^{6}}{8}\left(1+\sqrt{1+\left(\frac{r_{H}}{r}\right)^{4}}\right)^{3} d t^{2}+\frac{1+\sqrt{1+\left(\frac{r_{H}}{r}\right)^{4}}}{2\left(1+\left(\frac{r_{H}}{r}\right)^{4}\right)} \frac{d r^{2}}{r^{2}}+r^{2} d x^{2} .
$$

Note that to derive eq. (3.54), $t$ has been rescaled and the relation (3.43) has been used. As mentioned above, this solution is locally flat at the origin $r=0$, and asymptotically to the Lifshitz spacetime as $r \rightarrow \infty$ with $z=3$. The space-time in this region is complete and free of any kind of space-time curvature singularities. So, it represents a Lifshitz soliton [50-52]. 
On the other hand, in both of the ranges $r_{*} \in[-1,1]$ and $r \in[1, \infty)$, the space-time is singular at the spatial infinity $r=\infty$ (or $r_{*}=1$ ). Then, the physical interpretations of the solutions in these ranges are not clear (if there is any).

It is not difficult to convince oneself that the same is true for other choices of $s$ with $s \geq 1$.

\section{$3.2 .20<s<1$}

In this case, we find that

$$
r\left(r_{*}\right)= \begin{cases}r_{H}, & r_{*} \rightarrow-\infty, \\ 0, & r_{*}=-1, \\ \infty, & r_{*}=-s, \\ 0, & r_{*}=+1, \\ r_{H}, & r_{*} \rightarrow+\infty\end{cases}
$$

figure 2 shows the function $r\left(r_{*}\right)$ vs $r_{*}$, from which we can see that the region $r \in[0, \infty)$ is mapped into the region $r_{*} \in[-1,-s)$ or $r_{*} \in(-s,+1]$. The region $r_{*} \in(-\infty,-1]$ or $r_{*} \in[+1,+\infty)$ is mapped into the one $r \in\left[0, r_{H}\right)$. At the origin $r=0$, the metric takes the form (3.33) for $r_{*} \simeq-1$, and the form (3.30) for $r_{*} \simeq+1$. At $r_{*} \simeq-1$ the space-time is locally flat, while at $r_{*} \simeq+1$ it is singular. On the other hand, at the spatial infinity $(r \rightarrow \infty)$ (or $r_{*} \rightarrow-s$ ), it takes the Lifshitz form eq. (3.42) with $z=s$.

Note that, in the region $r_{*} \in[-1,-s)$, we have $\epsilon^{-}=\left.\operatorname{sign}\left(r_{*}+1\right)\right|_{r_{*} \simeq-1}=+1$. Then, the conditions (3.34) and (3.43) now require

$$
\Lambda>0,(0<s<1) .
$$

On the other hand, if we choose to work in the region $r_{*} \in(-s,+1]$, we find that $\epsilon^{+}=\left.\operatorname{sign}\left(r_{*}-1\right)\right|_{r_{*} \simeq+1}=-1$. Then, the conditions (3.31) and (3.43) require $\Lambda>0$, which is the same as that given by eq. (3.56). However, as pointed out above, the spacetime is locally flat at $r_{*}=-1$, while has a curvature singularity at $r_{*}=+1$. Moreover, since the metric coefficients are well-defined in this region, the singularity is naked.

Therefore, in the present case the solution in the region $r_{*} \in[-1,-s)($ or $r \in[0, \infty))$ represents the Lifshitz soliton [50-52], while in the region $r_{*} \in(-s, 1]$, which also corresponds to $r \in[0, \infty)$, the solution represents the Lifshitz space-time but with a curvature singularity located at $r=0$ (or $r_{*}=1$ ).

The spacetimes in the regions $r_{*} \in(-\infty,-1]$ and $r_{*} \in[1,+\infty)$ are incomplete, and extensions beyond $r_{*}= \pm \infty$ ( or $r=r_{H}$ ) are needed. As a representative example, let us consider the case $s=1 / 3$. Then, from eqs. (3.46) and (3.47) we find that,

$$
\begin{aligned}
\left(\frac{r}{r_{H}}\right)^{2} & =-\frac{\epsilon^{-}}{2} \mathfrak{R}\left(\epsilon^{+} \mathfrak{R}^{2}-3 \epsilon^{s}\right), \\
\Re & \equiv\left|\frac{r_{*}-1}{r_{*}+\frac{1}{3}}\right|^{1 / 2} .
\end{aligned}
$$

To study the solutions further, we consider it in each region marked in figure 2, separately. 
(a) In the region $r_{*} \in[-1,-1 / 3)$, we have $\epsilon^{+}=-\epsilon^{-}=\epsilon^{s}=-1$. Then, from eq. (3.46) we find that

$$
\mathfrak{R}(r)=\left[\left(\frac{r}{r_{H}}\right)^{2}+\sqrt{\left(\frac{r}{r_{H}}\right)^{4}-1}\right]^{-\frac{1}{3}}+\left[\left(\frac{r}{r_{H}}\right)^{2}+\sqrt{\left(\frac{r}{r_{H}}\right)^{4}-1}\right]^{\frac{1}{3}} .
$$

Note that the above expression is seemingly real only in the region $r \geq r_{H}$. However, a more careful study reveals that it is real for all $r \in(0, \infty)$. To see this, let us introduce $\theta$, defined via the relations,

$$
\cosh \theta=\left(\frac{r}{r_{H}}\right)^{2}, \quad \sinh \theta=\sqrt{\left(\frac{r}{r_{H}}\right)^{4}-1} .
$$

Then, in terms of $\theta$, we find that

$$
\mathfrak{R}(r)=2 \cosh \frac{\theta}{3} .
$$

From eq. (3.59) we can see that $\theta$ is well-defined even for $r<r_{H}$, for which it just becomes imaginary, but $\mathfrak{R}(r)$ is still well-defined and real. The only difference now is to replace $\cosh (\theta / 3)$ by $\cos (\bar{\theta} / 3)$, that is,

$$
\mathfrak{R}(r)=2 \cos \frac{\bar{\theta}}{3},\left(r<r_{H}\right),
$$

with

$$
\cos \bar{\theta}=\left(\frac{r}{r_{H}}\right)^{2}, \quad \sin \bar{\theta}=\sqrt{1-\left(\frac{r}{r_{H}}\right)^{4}},\left(r<r_{H}\right),
$$

where $\bar{\theta} \in[0, \pi / 2]$. Therefore, for any $r \in(0, \infty)$, eq. (3.58) is well-defined, and always real. It is smoothly crossing $r=r_{H}$, at which $\mathfrak{R}=2$ and $\bar{\theta}=0$. The origin $r=0$ corresponds to $\bar{\theta}=\pi / 2$, at which we have $\mathfrak{R}(\pi / 2)=\sqrt{3}$. In terms of $r$, the metric takes the form,

$$
d s^{2}=-r^{2 z} f^{2}(r) d t^{2}+\frac{g^{2}(r)}{r^{2}} d r^{2}+r^{2} d x^{2},
$$

where the functions $f$ and $g$ are given by,

$$
\begin{aligned}
f^{2} & =N_{0}^{2} r^{-2 z} \mathfrak{R}, \\
g^{2} & =\frac{2 \mathfrak{R}\left(\frac{r}{r_{H}}\right)^{2}}{1+2\left(\frac{r}{r_{H}}\right)^{2} \mathfrak{R}+\mathfrak{R}^{2}},
\end{aligned}
$$

with $\mathfrak{R} \geq \sqrt{3}$, as it can be seen from eqs. (3.60) and (3.61). At $r=0$ we have $\bar{\theta}=\pi / 2$ and $\mathfrak{R}=\sqrt{3}$. But, as shown above, this singularity is a coordinate one, and the space-time now is free of any kind of curvature singularities. So, it represents a Lifshitz soliton [50-52]. 
(b) In the region $r_{*} \in(-1 / 3,1]$, we have $-\epsilon^{+}=\epsilon^{-}=\epsilon^{s}=1$. Then, from eq. (3.46) we find

$$
\mathfrak{R}(r)=\left(\frac{r}{r_{H}}\right)^{\frac{2}{3}}\left\{\left[1-\sqrt{1+\left(\frac{r_{H}}{r}\right)^{4}}\right]^{\frac{1}{3}}+\left[1+\sqrt{1+\left(\frac{r_{H}}{r}\right)^{4}}\right]^{\frac{1}{3}}\right\},
$$

for which we have,

$$
\begin{aligned}
& f^{2}=N_{0}^{2} r^{-2 z} \mathfrak{R}, \\
& g^{2}=\frac{\left(\mathfrak{R}^{2}+2\right)\left(\mathfrak{R}^{2}-1\right)}{\left(1+\mathfrak{R}^{2}\right)^{2}} .
\end{aligned}
$$

Clearly, the functions $f$ and $g$ vanish at $\mathfrak{R}=0$ and $\mathfrak{R}=1$, respectively. To see the natures of these singularities, let us first note that in this region we have

$$
\begin{aligned}
\left(\frac{r}{r_{H}}\right)^{2} & =\frac{1}{2} \mathfrak{R}\left(\mathfrak{R}^{2}+3\right), \\
\mathfrak{R} & \equiv \sqrt{\frac{1-r_{*}}{r_{*}+\frac{1}{3}}},\left(-1 / 3 \leq r_{*} \leq 1\right) .
\end{aligned}
$$

Therefore, $\mathfrak{R}=0$ corresponds to $r=0$ (or $r_{*}=1$ ), at which the space-time is singular, as shown above. On the other hand, $\mathfrak{R}=1$ corresponds to $r=\sqrt{2} r_{H}$ (or $r_{*}=1 / 3$ ). This is a coordinate singularity, since in terms of $r_{*}$, the metric is well-defined at this point, as can be seen from eq. (3.27), which now reduces to,

$$
\begin{aligned}
d s^{2}= & -N_{0}^{2} \sqrt{\frac{1-r_{*}}{r_{*}+\frac{1}{3}}} d t^{2}+\frac{2 \gamma_{1}^{2} d r_{*}^{2}}{9 \beta \Lambda\left(r_{*}^{2}-1\right)\left(r_{*}+\frac{1}{3}\right)^{2}} \\
& +r_{H}^{2} \sqrt{\frac{1-r_{*}}{r_{*}+\frac{1}{3}}}\left(\frac{r_{*}+1}{r_{*}+\frac{1}{3}}\right) d^{2} x .
\end{aligned}
$$

Therefore, the space-time in this region represents a Lifshitz space-time, but now with a time-like singularity located at the origin $r=0$ (or $r_{*}=1$ ).

(c) In the region $r_{*} \in[1,+\infty)$, we have $\epsilon^{+}=\epsilon^{-}=\epsilon^{s}=1$. Then, from eq. (3.46) we find,

$$
2\left(\frac{r}{r_{H}}\right)^{2}=-\mathfrak{R}\left(\mathfrak{R}^{2}-3\right),
$$

which in general has three real roots for $r<r_{H}$. In fact, introducing the angle $\bar{\theta}$ as defined by eq. (3.62), the three roots can be written in the form,

$$
\mathfrak{R}_{k}=2 \cos \frac{(2 k+1) \pi+\bar{\theta}}{3}, \quad k=0, \pm 1 .
$$

Since $\mathfrak{R} \geq 0$ in the region $r \leq r_{H}$, it can be seen that only $\mathfrak{R}_{0}$ and $\mathfrak{R}_{-1}$ satisfy this condition. However, with $\mathfrak{R}=\mathfrak{R}_{-1}$, we find that $\mathfrak{R} \in[1, \sqrt{3}]$, which leads to $r_{*} \in(-\infty,-1]$, as now we have

$$
r_{*}=\frac{1}{3}\left(\frac{4}{1-\mathfrak{R}^{2}}-1\right) .
$$


On the other hand, for $\mathfrak{R}=\mathfrak{R}_{0}$, we find that $\mathfrak{R} \in[0,1]$ and $r_{*} \in[1,+\infty)$. Therefore, $\mathfrak{R}_{0}$ is the solution we are looking for. With this root, the metric takes the form of eq. (3.63), but now the functions $f$ and $g$ are given by

$$
\begin{aligned}
f^{2} & =N_{0}^{2} r^{-2 z} \mathfrak{R}, \\
g^{2} & =\frac{2 \mathfrak{R}\left(\frac{r}{r_{H}}\right)^{2}}{1+2\left(\frac{r}{r_{H}}\right)^{2} \mathfrak{R}+\mathfrak{R}^{2}} .
\end{aligned}
$$

It must be noted that $\mathfrak{R}_{0}$ becomes complex when $r>r_{H}$. Therefore, simply taking $r>r_{H}$ in the above expressions will result in complex metric coefficients, and cannot be considered as a viable extension of the solution to the region $r>r_{H}$.

On the other hand, the root $\mathfrak{R}_{+1}[=-2 \cos (\bar{\theta} / 3)]$ is real in both of the regions $r \geq r_{H}$ and $r \leq r_{H}$. In particular, for $r>r_{H}$, it takes the form,

$$
\mathfrak{R}_{+1}=-\left[\left(\frac{r}{r_{H}}\right)^{2}-\sqrt{\left(\frac{r}{r_{H}}\right)^{4}-1}\right]^{-\frac{1}{3}}-\left[\left(\frac{r}{r_{H}}\right)^{2}-\sqrt{\left(\frac{r}{r_{H}}\right)^{4}-1}\right]^{\frac{1}{3}} .
$$

However, for this root we have $\mathfrak{R} \in[-2,-\sqrt{3}]$, which is not allowed by eq. (3.47).

(d) In the region $r_{*} \in(-\infty,-1]$, we have $\epsilon^{+}=\epsilon^{-}=\epsilon^{s}=-1$. Then, $\mathfrak{R}$ satisfies the same equation (3.69), which for $r<r_{H}$ has the three real roots, given by eq. (3.70). However, as shown above, only the one

$$
\mathfrak{R}=2 \cos \frac{\pi-\bar{\theta}}{3},
$$

corresponds to $r_{*} \in(-\infty,-1)$. The functions $f$ and $g$ are the same as those given by eq. (3.72).

\subsection{3 $-1<s<0$}

In this case, we find that

$$
r\left(r_{*}\right)= \begin{cases}r_{H}, & r_{*} \rightarrow-\infty, \\ 0, & r_{*}=-1, \\ \infty, & r_{*}=|s|, \\ 0, & r_{*}=+1, \\ r_{H}, & r_{*} \rightarrow+\infty .\end{cases}
$$

figure 3 shows the function $r\left(r_{*}\right)$ vs $r_{*}$. The space-time near the points $r_{*}= \pm 1$ and $r_{*}=-s$ have similar behavior, at which the metric is given, respectively, by eq. (3.30), (3.33) and (3.42). As a result, the singularity at $r_{*}=1(r=0)$ is a scalar one and naked, while at 


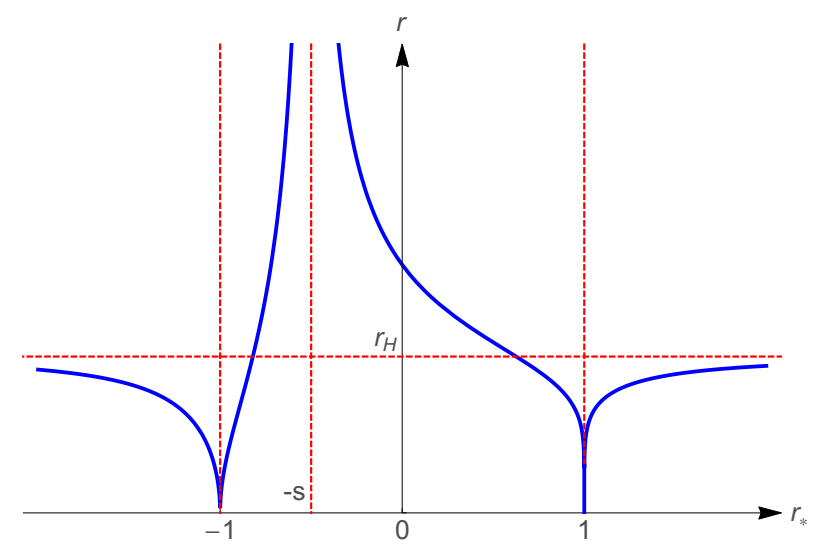

Figure 2. The function $r \equiv r_{+}\left(r_{*}\right)$ defined by eq. (3.22) vs $r_{*}$ for $0<s<1$. The space-time is singular at $r_{*}=1$, locally flat at $r_{*}=-1$, and asymptotically to the Lifshitz space-time (3.15) with $z=s$ as $r_{*} \rightarrow-s(r \rightarrow \infty)$.

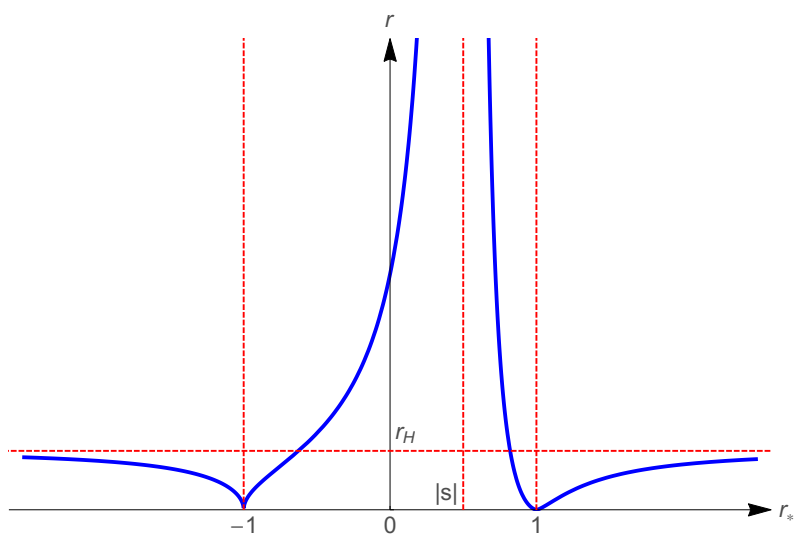

Figure 3. The function $r \equiv r_{+}\left(r_{*}\right)$ defined by eq. (3.22) vs $r_{*}$ for $-1<s<0$. The space-time is singular at $r_{*}=+1$, locally flat at $r_{*}=-1$, and asymptotically to the Lifshitz space-time (3.15) with $z=s$ as $r_{*} \rightarrow|s|(r \rightarrow \infty)$.

$r_{*}=-1(r=0)$ it is locally flat. As $r_{*} \rightarrow|s|($ or $r \rightarrow \infty)$ it is asymptotically Lifshitz spacetime with $z=s$, that is, $-1<z<0$. Since now we have $\epsilon^{-}=\left.\operatorname{sign}\left(r_{*}+1\right)\right|_{r_{*} \simeq|s|}=+1$. Then, in the region $r_{*} \in[-1,|s|)$, the conditions (3.34) and (3.43) now require

$$
\Lambda>0,(-1<s<0) .
$$

On the other hand, if we choose to work in the region $r_{*} \in(|s|,+1]$, we find that near $r=1$ we have $\epsilon^{+}=\left.\operatorname{sign}\left(r_{*}-1\right)\right|_{r_{*} \simeq 1}=-1$. Then, the conditions (3.31) and (3.43) also require eq. (3.76) to be held, although now the space-time has a curvature singularity at $r_{*}=1(r=0)$.

In review of the above solutions, it is remarkable to note that a positive cosmological constant always produces an asymptotically Lifshitz space-time with the anisotropic scaling exponent $z$ less than one, while a negative cosmological constant always produces an asymptotically Lifshitz space-time with the anisotropic scaling exponent $z$ greater than 
one, that is,

$$
z= \begin{cases}<1, & \Lambda>0 \\ >1, & \Lambda<0\end{cases}
$$

Similar to the previous cases, let us consider the case with $s=-1 / 3$ in detail. Then, we find that

$$
\begin{aligned}
\left(\frac{r}{r_{H}}\right)^{2} & =\mathfrak{R}\left|r_{*}+1\right|\left|r_{*}-\frac{1}{3}\right|^{1 / 2}, \\
\mathfrak{R} & =\left(\frac{r_{*}-1}{r_{*}-\frac{1}{3}}\right)^{2} .
\end{aligned}
$$

Following what we did for the cases $s=3$ and $s=1 / 3$, one can solve it for $\mathfrak{R}$ in the following four regions.

(a) $r_{*} \in[-1,1 / 3)$. In this region, we have the following solution

$$
\mathfrak{R}^{\frac{1}{2}}=-\frac{1}{2}+\frac{1}{2}\left[\frac{r}{r_{H}}+\sqrt{1+\left(\frac{r}{r_{H}}\right)^{2}}\right]^{-\frac{2}{3}}+\frac{1}{2}\left[\frac{r}{r_{H}}+\sqrt{1+\left(\frac{r}{r_{H}}\right)^{2}}\right]^{\frac{2}{3}} .
$$

Then, the functions $f$ and $g$ are given by

$$
f^{2}=N_{0}^{2} r^{-2 z} \mathfrak{R}^{-\frac{1}{2}}, \quad g^{2}=\frac{2 \Re-3 \mathfrak{R}^{\frac{1}{2}}}{2\left(1-\mathfrak{R}^{\frac{1}{2}}\right)^{2}},
$$

where we had used the relation

$$
\mathfrak{R}=\left(\frac{r_{*}-1}{r_{*}-\frac{1}{3}}\right)^{2}= \begin{cases}\frac{9}{4}, & r_{*}=-1, \\ \infty, & r_{*}=\frac{1}{3} .\end{cases}
$$

From the above expressions one can see that when $\mathfrak{R}=9 / 4$ (corresponding to $r=0$ ) the function $g$ is vanishing. At this point, we have $r_{*}=-1$ which is not a curvature singularity as what we had proved in the previous section. In fact, the space-time in the present case is free of any kind of space-time curvature singularity, and represents a Lifshitz soliton.

(b) $r_{*} \in(1 / 3,1] . \mathfrak{R}$ in this region is given by

$$
\mathfrak{R}^{\frac{1}{2}}= \begin{cases}-\frac{1}{2}+\frac{1}{2} \mathcal{A}(r)^{-\frac{2}{3}}+\frac{1}{2} \mathcal{A}(r)^{\frac{2}{3}}, & r \geq r_{H}, \\ -\frac{1}{2}+\cos \frac{2 \tilde{\theta}}{3}, & r<r_{H},\end{cases}
$$

where we have defined

$$
\mathcal{A}(r)=\frac{r}{r_{H}}+\sqrt{\left(\frac{r}{r_{H}}\right)^{2}-1}
$$


with $\tilde{\theta}$ being given by

$$
\cos \tilde{\theta}=\frac{r}{r_{H}}, \quad \sin \tilde{\theta}=\sqrt{1-\left(\frac{r}{r_{H}}\right)^{2}} .
$$

The functions $f$ and $g$ are given by

$$
f^{2}=N_{0}^{2} r^{-2 z} \mathfrak{R}^{-\frac{1}{2}}, \quad g^{2}=\frac{2 \mathfrak{R}+5 \Re^{\frac{1}{2}}}{2\left(1+\mathfrak{R}^{\frac{1}{2}}\right)^{2}} .
$$

Note that the metric coefficients are well-defined along the whole real axis $r \in(0, \infty)$, except at the origin $r=0$ (or $r_{*}=1$ ), which corresponds to $\mathfrak{R}=0$. As shown above, this represents a real space-time curvature singularity. Therefore, the solution in this case represents a Lifshitz spacetime with a curvature singularity at $r=0$.

(c) $r_{*} \in(1,+\infty)$. In this region $\mathfrak{R}$ is given by

$$
\mathfrak{R}=\frac{1}{2}+\cos \frac{2 \tilde{\theta}+\pi}{3}= \begin{cases}1, & r=r_{H}, \\ 0, & r=0,\end{cases}
$$

where $\tilde{\theta}$ is defined by eq. (3.84), so that $\mathfrak{R} \in(0,1)$. Then, the functions $f$ and $g$ are given by

$$
f^{2}=N_{0}^{2} r^{-2 z} \mathfrak{R}^{-\frac{1}{2}}, \quad g^{2}=\frac{2 \mathfrak{R}-3 \mathfrak{R}^{\frac{1}{2}}}{2\left(1-\mathfrak{R}^{\frac{1}{2}}\right)^{2}} .
$$

Clearly, the metric becomes singular at $r=r_{H}$. But, this singularity is a coordinate one and extension beyond this surface is needed. Simply assuming that eq. (3.84) holds also for $r>r_{H}$ will lead to $\mathfrak{R}$ to be a complex function of $r$, and so are the functions $f$ and $g$. Therefore, this will not represent a desirable extension.

(d) $r_{*} \in(-\infty,-1]$. Similar to the region $r_{*} \in(1,+\infty)$, in the present case we have

$$
\mathfrak{R}=\frac{1}{2}+\cos \frac{2 \tilde{\theta}}{3}= \begin{cases}\frac{3}{2}, & r=r_{H}, \\ 1, & r=0 .\end{cases}
$$

Since $\tilde{\theta} \in[0, \pi / 2]$, we have $\mathfrak{R} \geq 1$ for $r \in\left[0, r_{H}\right]$. The functions $f$ and $g$ are also given by eq. (3.87), from which we can see that $g$ becomes unbounded at $r=0$ (or $\left.r_{*}=-1\right)$. As shown above, this is a coordinate singularity.

To extend the above solution to the region $r>r_{H}$, one may simply assume that eq. (3.84) hold also for $r>r_{H}$. In particular, setting $\tilde{\theta}=i \hat{\theta}$, we find that

$$
\mathfrak{R}=\frac{1}{2}+\cosh \frac{2 \hat{\theta}}{3} \geq \frac{3}{2},\left(r \geq r_{H}\right),
$$


where $\hat{\theta}$ is defined by

$$
\cosh \hat{\theta}=\left(\frac{r}{r_{H}}\right), \quad \sinh \hat{\theta}=\sqrt{\left(\frac{r}{r_{H}}\right)^{2}-1} .
$$

The above represents an extension of the solution originally defined only for $r \leq r_{H}$. Note that $\mathfrak{R} \simeq r^{4 / 3}$ as $r \rightarrow \infty$. Then, from eq. (3.87) we find that

$$
r^{2 z} f^{2} \sim r^{-2 / 3}, \quad g^{2} \simeq 1
$$

as $r \rightarrow \infty$. That is, the space-time is asymptotically approaching to a Lifshitz spacetime with its dynamical exponent now given by $z=-1 / 3$. But, at the origin $r=0$ ( or $r_{*}=-1$ ), the space-time is free of any kind of space-time curvature singularity. Therefore, the extended solution represents a Lifshitz soliton.

\subsection{4 $s<-1$}

In this case, we find that

$$
r\left(r_{*}\right)= \begin{cases}r_{H}, & r_{*} \rightarrow-\infty \\ 0, & r_{*}=-1 \\ \infty, & r_{*}=+1 \\ 0, & r_{*}=|s| \\ r_{H}, & r_{*} \rightarrow+\infty\end{cases}
$$

figure 4 shows the function $r\left(r_{*}\right)$ vs $r_{*}$, from which we can see that the space-time is singular at the spatial infinity $r=\infty$ (or $r_{*}=+1$ ). Then, it is not clear whether the space-time in the region $r_{*} \in[-1,+1]$ represents any physical reality. However, in the regions $r_{*} \in(-\infty,-1]$ and $r_{*} \in[1,+\infty)$ they may represent the interns of Lifshitz black holes. To see this explicitly, we take $s=-3$ as a specific example. Just follows what we have done in the previous subsections. In the region $r \in(s,-1]$, from eq. (3.46) we can obtain the functions $f$ and $g$

$$
\begin{aligned}
f^{2} & =N_{0}^{2} r^{-2 z}\left(\frac{r}{r_{H}}\right)^{6}\left(1+\sqrt{1-\left(\frac{r}{r_{H}}\right)^{2}}\right)^{3}, \\
g^{2} & =\frac{1-\sqrt{1-\left(\frac{r}{r_{H}}\right)^{2}}}{2\left(1-\left(\frac{r}{r_{H}}\right)^{2}\right)}
\end{aligned}
$$

This solution is only well defined in the region $r \in\left[0, r_{H}\right]$.

On the other hand, if we focus on the region $r \in[-1,1]$, which may physically be viewed as a Lifshitz soliton. To see this clearly, we solve eq. (3.46) and obtain the following 


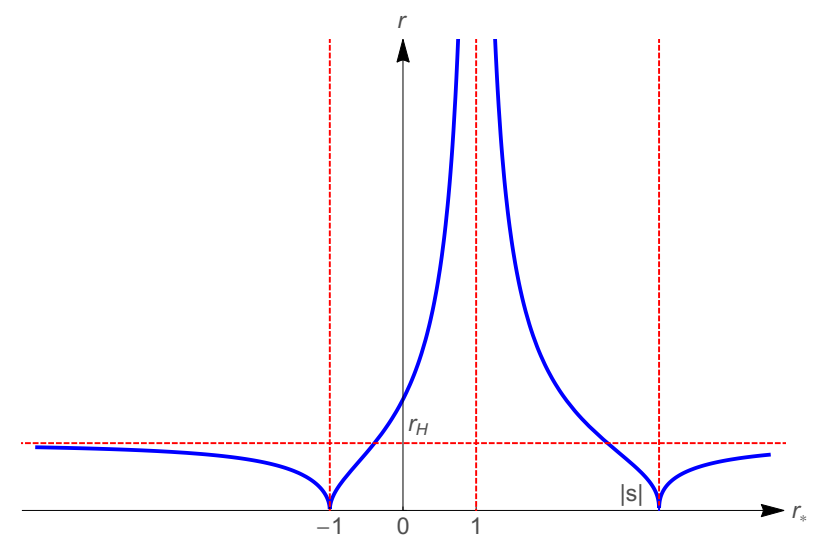

Figure 4. The function $r \equiv r_{+}\left(r_{*}\right)$ defined by eq. (3.22) vs $r_{*}$ for $s<-1$. The space-time is singular at $r_{*}=+1(r=\infty)$.

expressions

$$
\begin{aligned}
f^{2} & =N_{0}^{2} r^{-2 z}\left(\frac{r}{r_{H}}\right)^{6}\left(\sqrt{1+\left(\frac{r}{r_{H}}\right)^{2}}-1\right)^{3}, \\
g^{2} & =\frac{1-\sqrt{1+\left(\frac{r}{r_{H}}\right)^{2}}}{2\left(1+\left(\frac{r}{r_{H}}\right)^{2}\right)}
\end{aligned}
$$

It should be noted that the above analysis is not valid for $s=0, \pm 1$, as one can see from eqs. (3.11), (3.21) and (3.25). In the following, let us consider these particular cases, separately.

\subsection{Generalized BTZ black holes}

When $s=1$, from eq. (3.11) we find that $\beta=0$, which leads to $c_{s}$ to become unbounded unless $\lambda=1$, as can be seen from eq. (2.20). This corresponds to the relativistic limit that requires $\left(\beta, \lambda, \gamma_{1}\right)=(0,1,-1)$. These values are protected by the symmetry (general covariance) of the theory, and they remain the same even after radiative corrections are taken into account. In this limit, the spin-0 gravitons disappear, and the corresponding gravity is purely topological [94]. Nevertheless, the theory still provides valuable information on various important issues, such as black holes [98]. In the HL gravity, the general covariance is replaced by the foliation-preserving diffeomorphisms, and in principle these parameters now can take any values, when radiative corrections are taken into account. However, as shown in the last section, the stability and ghost-free conditions in the IR limit require $\lambda=1$ when $\beta=0$. Therefore, in the rest of the paper, we shall assume that $\lambda=1$ whenever $\beta=0$.

When $s=1$, eq. (3.10) becomes invalid, and nor is eq. (3.12). Then, we must come back to the original equations (3.8) and (3.9), which now become,

$$
\begin{array}{r}
\gamma_{1}\left(r g^{\prime}-g\right)+\Lambda g^{3}=0 \\
\gamma_{1} W-\Lambda g^{2}=0
\end{array}
$$


and have the general solutions,

$$
g^{2}=\frac{\gamma_{1} r^{2}}{M+\Lambda r^{2}}, \quad f^{2}=f_{0}^{2} \frac{\left|M+\Lambda r^{2}\right|}{r^{2 z}},
$$

where $M$ and $f_{0}$ are the integration constants. By rescaling $t$, without loss of the generality, we can always set $f_{0}=1$, and the metric takes the form,

$$
d s^{2}=-\left|M \pm\left(\frac{r}{\ell}\right)^{2}\right| d t^{2}+\left(\frac{\gamma_{1}}{M \pm\left(\frac{r}{\ell}\right)^{2}}\right) d r^{2}+r^{2} d x^{2}
$$

where "+" ("-") corresponds to $\Lambda>0(\Lambda<0)$, and $\ell \equiv 1 / \sqrt{|\Lambda|}$. Clearly, to have $g_{r r}$ non-negative, we must require

$$
M \pm\left(\frac{r}{\ell}\right)^{2}= \begin{cases}\geq 0, & \gamma_{1}>0 \\ \leq 0, & \gamma_{1}<0\end{cases}
$$

The BTZ black hole solution [98] corresponds to $\left(\lambda, \gamma_{1}\right)=(1,-1)$ and $\Lambda<0$, for which the corresponding action becomes generally covariant, and the constant $M$ denotes the mass of the BTZ black hole.

It is interesting to note that black holes with $\Lambda<0$ exist for any given $\gamma_{1}$. Then, we refer them to as the generalized BTZ black holes.

\subsection{Solutions with $s=-1$}

When $s=-1$, from eq. (3.11) we find that $\beta=2 \gamma_{1}$. Then, for $W=W_{+}$eq. (3.12) becomes,

$$
2 r r_{*}^{\prime}-\left(r_{*}^{2}-1\right)\left(r_{*}-1\right)=0
$$

which has the solution,

$$
r_{+}\left(r_{*}\right)=r_{H}\left|\frac{r_{*}+1}{r_{*}-1}\right|^{1 / 2} e^{-\frac{1}{r_{*}-1}}
$$

where $r_{H}$ is a constant. It can be shown that the corresponding functions $g$ and $f$ are given by

$$
\begin{aligned}
f\left(r_{*}\right) & =f_{0}\left|\frac{r_{*}-1}{r_{*}+1}\right|^{z / 2} e^{\frac{1+z}{r_{*}-1}}, \\
g^{2}\left(r_{*}\right) & =\frac{\gamma_{1}}{4 \Lambda}\left(r_{*}^{2}-1\right) .
\end{aligned}
$$

By properly rescaling the coordinates $t$ and $x$, the corresponding line element can be cast in the form,

$$
d s^{2}=-e^{\frac{2}{r_{*}-1}} d t^{2}+\left(\frac{\gamma_{1}}{\Lambda}\right) \frac{d r_{*}^{2}}{\left(r_{*}^{2}-1\right)\left(r_{*}-1\right)^{2}}+\left|\frac{r_{*}+1}{r_{*}-1}\right| e^{-\frac{2}{r_{*}-1}} d^{2} x .
$$

Note that the functions $g\left(r_{*}\right)$ and $f\left(r_{*}\right)$ given by eq. (3.102) are well-defined even for $r_{*}<0$, although according to eq. (3.11) it is non-negative. Therefore, similar to the previous cases, 


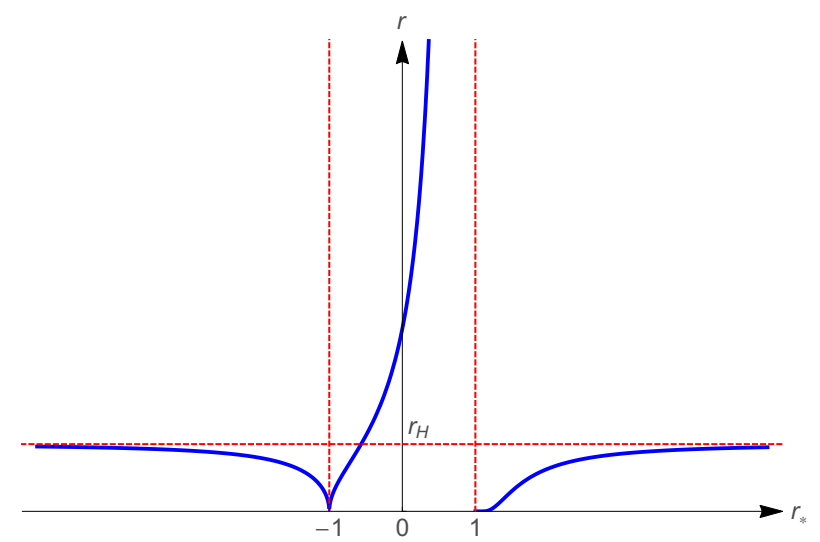

Figure 5. The function $r \equiv r_{+}\left(r_{*}\right)$ defined by eq. (3.101) vs $r_{*}$ for $s=-1$. The spacetime is singular at $r_{*}= \pm 1$.

we consider the region $r_{*}<0$ as a natural extension, and consider spacetimes defined over the whole region $r_{*} \in(-\infty,+\infty)$.

In addition, in this particular case, $r_{*}$ is dimensionless, while $x$ has the dimension of length, as one can see from eq. (3.103). From eq. (3.101), we find that

$$
r\left(r_{*}\right)= \begin{cases}r_{H}, & r_{*} \rightarrow-\infty \\ 0, & r_{*}=-1, \\ \infty, & r_{*} \rightarrow 1^{-} \\ 0, & r_{*} \rightarrow 1^{+} \\ r_{H}, & r_{*} \rightarrow+\infty\end{cases}
$$

figure 5 shows the curve of $r$ vs $r_{*}$. The space-time is singular at $r_{*}= \pm 1$, as one can see from the corresponding Ricci scalar, given by

$$
R=\frac{32 \Lambda^{2}\left(r_{*}^{2}-r_{*}-1\right)}{\gamma_{1}^{2}\left(r_{*}^{2}-1\right)^{2}} .
$$

Therefore, one may restrict the space-time to the region $r_{*} \in(1, \infty)$ or $r_{*} \in(-\infty,-1)$. In each of these two regions, to have a proper sign of the metric, we must require $\gamma_{1} / \Lambda<0$, as one can see from eq. (3.103). However, as $\left|r_{*}\right| \rightarrow \infty$ we always have $r \rightarrow r_{H}$ (finite). So, to have a complete space-time, extension of the solutions to the region $r>r_{H}$ is needed.

It can be shown that the solution with the choice $W=W_{-}$can be also obtained from the one of $W=W_{+}$by replacing $r_{*}$ by $-r_{*}$. So, in the following we shall not consider it.

\subsection{Solutions with $s=0$}

When $s=0$ from eq. (3.11) we have $\gamma_{1}=0$. Then, the function $r_{*}$ defined there becomes unbound, and eq. (3.12) is no longer valid. In fact, when $\gamma_{1}=0$, from eq. (3.9) we find that

$$
W=\alpha g,
$$


where $\alpha \equiv \sqrt{2 \Lambda / \beta}$. Inserting it into eq. (3.8) we obtain

$$
\alpha g(r)=0 .
$$

Since $g \neq 0$, we must have $\alpha=0$ or $\Lambda=0$. Then, the function $g(r)$ is undetermined. On the other hand, from eqs. (3.5) and (3.106) we find that

$$
f=\frac{f_{0}}{r^{z}}
$$

where $f_{0}$ is a constant. By rescaling $t$, one can always set it to one. Thus, in this case the metric takes the form,

$$
d s^{2}=-d t^{2}+\frac{g^{2}(r) d r^{2}}{r^{2}}+r^{2} d x^{2}
$$

where $g$ is an arbitrary function of $r$, and $\Lambda=0$. Setting

$$
r_{*}=\int \frac{g(r) d r}{r}+r_{*}^{0}
$$

where $r_{*}^{0}$ is a constant, the above metric takes the form,

$$
d s^{2}=-d t^{2}+d r_{*}^{2}+r^{2}\left(r_{*}\right) d x^{2},
$$

where $r\left(r_{*}\right)$ is an arbitrary function of $r_{*}$.

\section{Static vacuum solutions for the non-diagonal case $\boldsymbol{N}^{r} \neq 0$}

When $N^{r} \neq 0$, it is found convenient to consider solutions with $\lambda=1$ and the ones with $\lambda \neq 1$, separately.

\subsection{Solutions with $\lambda=1$}

In this subcase, the Hamiltonian constraint (3.3), the momentum constraint (3.4) and the dynamical equation (3.6) reduce, respectively, to

$$
\begin{aligned}
\frac{H}{r^{2 z} f^{2}}\left(\frac{H}{r}\right)^{\prime}+\beta\left[\frac{\left(r^{z} f W\right)^{\prime}}{r^{z-1} f}+\frac{1}{2} W^{2}\right]+\gamma_{1} W-\Lambda g^{2} & =0 \\
\left(r^{z-1} g f\right)^{\prime} & =0 \\
\frac{H}{r^{2 z} f^{2}}\left(\frac{H}{r}\right)^{\prime}+\frac{\beta}{2} W^{2}+\gamma_{1} W-\Lambda g^{2} & =0 .
\end{aligned}
$$

From eq. (4.2) we find that

$$
g=\frac{g_{0}}{r^{z-1} f}=\frac{g_{0} r}{N}
$$

where $g_{0}$ is an integration constant. On the other hand, the combination of eqs. (4.1) and (4.3) yields,

$$
\beta\left(r^{z} f W\right)^{\prime}=0 .
$$

Thus, depending on whether $\beta$ vanishes or not, we obtain two different classes of solutions. 


\subsection{1 $\beta=0$}

As mentioned above, $\beta=0$ is allowed when $\lambda=1$. Then, eq. (4.5) holds identically, while eq. (4.1) reduces to eq. (4.3). Hence, now there are only two independent equations, (4.2) and (4.3), for three unknowns, $f(r), g(r)$ and $N^{r}(r)$. Therefore, in the present case the system is underdetermined. Taking $N^{r}(r)$ as arbitrary, from eq. (4.3) we find that

$$
\left(\frac{g N^{r}}{r}\right)^{2}=g_{0}^{2}\left(M+\Lambda r^{2}\right)-\gamma_{1} N^{2}
$$

where $M$ is a constant. Inserting eq. (4.4) into the above expression, we find that

$$
N^{2}=\frac{g_{0}^{2}}{2 \gamma_{1}}\left[\left(M+\Lambda r^{2}\right) \pm \sqrt{\left(M+\Lambda r^{2}\right)-4 \gamma_{1}\left(\frac{N^{r}}{g_{0}}\right)^{2}}\right] .
$$

Without loss of the generality, we can always set $g_{0}=1$, by rescaling $t \rightarrow g_{0} t$ and $N^{r}=$ $g_{0} \bar{N}^{r}$, so that the metric can be finally cast in the form,

$$
d s^{2}=-N^{2} d t^{2}+\frac{1}{N^{2}}\left(d r+N^{r} d t\right)^{2}+r^{2} d x^{2},
$$

where $N^{2}$ is given by eq. (4.7) with $g_{0}=1$. When $N^{r}=0$, the above metric reduces to the generalized BTZ solutions (3.98). When $N^{r} \neq 0$, the corresponding solutions can be considered as a further generalization of the BTZ solution [98].

To understand the question of the underdetermination of the system in the current case, it is suggestive to consider the diagonal metric

$$
d s^{2}=-e^{2 \Psi(r)} d \tau^{2}+e^{-2 \Psi(r)} d r^{2}+r^{2} d x^{2} .
$$

Then, setting

$$
\tau=t-\Sigma(r),
$$

where $\Sigma(r)$ is an arbitrary function, we find that in terms of $t$, the above metric takes the form,

$$
d s^{2}=-e^{2 \Psi(r)} d t^{2}+2 \Sigma^{\prime} e^{2 \Psi(r)} d t d r+\left(e^{-2 \Psi}-\Sigma^{\prime 2} e^{2 \Psi}\right) d r^{2}+r^{2} d x^{2} .
$$

Therefore, for any given diagonal solution $\Psi(r)$, we can always obtain a non-diagonal one $(\Psi, \Sigma)$ by the coordinate transformation (4.10), where $\Sigma$ is an arbitrary function of $r$, as mentioned above. Identifying the two metrics (4.8) and (4.11), we obtain

$$
\begin{aligned}
e^{2 \Psi} & =N^{2}-\left(\frac{N^{r}}{N}\right)^{2}, \\
\Sigma^{\prime} & =\frac{N^{r}}{N^{4}-\left(N^{r}\right)^{2}} .
\end{aligned}
$$

Therefore, the underdetermination of the system can be considered as due to the "free coordinate transformations" (4.10). However, in the HL theory, the symmetry (2.1) in general does not allow such transformations. If it is forced to do so, the resulted solutions usually do not satisfy the corresponding HL field equations. Examples of this kind were provided in [99]. However, it can be shown that the current case is an exception. 


\subsection{2 $\beta \neq 0$}

Then, eq. (4.5) yields

$$
\left(r^{z} f W\right)^{\prime}=0 .
$$

It is found convenient to consider the cases $W=0$ and $W \neq 0$, separately.

Case A.2.1) Solutions with $\boldsymbol{W}=\mathbf{0}$ : in this case, from eqs. (3.5) and the definition of $W$ we find that

$$
f=f_{0} r^{-z},
$$

where $f_{0}$ is a constant. Substituting it into eqs. (4.4) and (4.3) we find that

$$
\begin{aligned}
g & =g_{0} r \\
H & = \pm g_{0} f_{0} r \sqrt{1+M+\Lambda r^{2}},
\end{aligned}
$$

where $g_{0} \equiv C_{0} / f_{0}$, and $M$ is another integration constant. Then, we find that

$$
N^{r}= \pm f_{0} \sqrt{1+M+\Lambda r^{2}} \text {. }
$$

Rescaling the coordinates $t, r$ and $x$, without loss of then generality, we can set $f_{0}=$ $g_{0}=1$, so the corresponding metric of the solution finally takes the form,

$$
d s^{2}=-d t^{2}+\left(d r^{2}+\sqrt{1+M+\Lambda r^{2}} d t\right)^{2}+r^{2} d x^{2},
$$

which is nothing but the BTZ solution written in the Painleve-Gullstrand coordinates [100, 101], where $M$ denotes the mass of the BTZ black hole. Note that in writing the above metric, we had chosen the "+" sign of $N^{r}$. The corresponding metric for the choice of "_" sign can be trivially obtained by simply flipping the sign of $t$. Therefore, in the following we shall always choose its "+" sign, whenever the possibility raises.

Case A.2.2) Solutions with $\boldsymbol{W} \neq \mathbf{0}$ : then, eqs. (3.5) and (4.14) yield,

$$
f=f_{0} r^{-z}\left(\ln \frac{r}{r_{H}}\right), \quad W=\left(\ln \frac{r}{r_{H}}\right)^{-1},
$$

where $f_{0}$ and $r_{H}$ are two integration constants. Then, from eqs. (4.3) and (4.4) we find that

$$
\begin{aligned}
g & =g_{0} r\left(\ln \frac{r}{r_{H}}\right)^{-1}, \quad H=f_{0} r \mathcal{H}, \\
N^{r} & =\frac{f_{0}}{g_{0}} \mathcal{H} \ln \left(\frac{r}{r_{H}}\right),
\end{aligned}
$$

where

$$
\mathcal{H} \equiv\left[B-\beta \ln \left(\frac{r}{r_{H}}\right)-\gamma_{1} \ln ^{2}\left(\frac{r}{r_{H}}\right)+g_{0}^{2} \Lambda r^{2}\right]^{1 / 2}
$$

with $B$ being another integration constant. By rescaling the coordinates, we can always set $f_{0}=g_{0}=1$, and the metric takes the form,

$$
d s^{2}=-\ln ^{2}\left(\frac{r}{r_{H}}\right) d t^{2}+\frac{1}{\ln ^{2}\left(\frac{r}{r_{H}}\right)}\left[d r+\mathcal{H} \ln \left(\frac{r}{r_{H}}\right) d t\right]^{2}+r^{2} d x^{2} .
$$


Clearly, the metric becomes singular at $r=r_{H}$. To see the nature of the singularity, let us consider the qantities $K$ and $R$, which are given by

$$
\begin{aligned}
K & =\frac{\mathcal{H}}{r}\left[1-\frac{\beta+2 \gamma_{1} \ln \left(\frac{r}{r_{H}}\right)-2 \Lambda r^{2}}{2 \mathcal{H}^{2}}\right], \\
R & =-\frac{2}{r^{2}} \ln \left(\frac{r}{r_{H}}\right),
\end{aligned}
$$

which are finite at $r=r_{H}$, and indicate that the singularity at $r=r_{H}$ is a coordinate one.

On the other hand, to have the metric real, we must assume $\mathcal{H} \geq 0$, where

$$
\mathcal{H}= \begin{cases}\sqrt{B+\Lambda r_{H}^{2}}, & r=r_{H}, \\ \sqrt{\Lambda} r, & r \gg r_{H} .\end{cases}
$$

Clearly, we must assume $\Lambda \geq 0$ and $B \geq-\Lambda r_{H}^{2}$. Otherwise, $\mathcal{H}$ will becomes negative for $r>r_{\infty}$, where $r_{\infty}$ is a root of $\mathcal{H}(r)=0$, at which the spacetime becomes singular, as one can see from eq. (4.23). An interesting case is where $\Lambda=0$. Since $\beta<0$, we find that the condition $\mathcal{H}>0$ always holds for $B>0$ and $\gamma_{1}<0$. In this case, eq. (4.23) shows that the spacetime is also asymptotically flat as $r \rightarrow \infty$.

\subsection{Solutions with $\lambda \neq 1$}

When $\lambda \neq 1$, from the Hamiltonian constraint (3.3) and the dynamical equation (3.6) we obtain

$$
\beta\left[g r\left(\frac{W}{g}\right)^{\prime}+W\right]-\gamma_{1}\left(r \frac{g^{\prime}}{g}-1+W\right)=0 .
$$

To solve the above equations, let us consider some representative cases.

\subsection{1 $W=0$}

In this case, from eqs. (3.5) and (4.25) we find that

$$
f=f_{0} r^{-z}, \quad g=g_{0} r
$$

Substituting them into the momentum constraint (3.4), we find

$$
H=H_{0} r^{2}+H_{1}
$$

where $H_{0}$ and $H_{1}$ are two constants, which can be determined by the dynamical equation (3.6),

$$
H_{0}=\sqrt{\frac{\Lambda}{2 \lambda-1}} f_{0} g_{0}, \quad H_{1}=0
$$

Then, we find that

$$
N^{r}=\sqrt{\frac{\Lambda}{2 \lambda-1}} f_{0} r
$$


It can be shown that we can awlays set $f_{0}=g_{0}=1$ by resacling the cooridinates, so that the metric can be written in the form,

$$
d s^{2}=-d t^{2}+\left(d r+\sqrt{\frac{\Lambda r^{2}}{2 \lambda-1}} d t\right)^{2}+r^{2} d x^{2},
$$

which is the BTZ solution written in the Painleve-Gullstrand cooridnates, with

$$
\Lambda_{\text {eff. }} \equiv \frac{\Lambda}{2 \lambda-1}, \quad M=-1 .
$$

That is, the corresponding mass is negative in the current case.

\subsection{2 $W=z$}

In this case, it can be shown that the functions $f$ and $g$ are all constants, provided that $z$ satisfies the relation,

$$
z=s=\frac{\gamma_{1}}{\gamma_{1}-\beta} .
$$

Without loss of the generality, we set $f=g=1$, so that $N^{r}=H$, and the corresponding metric takes the form,

$$
d s^{2}=-r^{2 z} d t^{2}+\frac{1}{r^{2}}(d r+H d t)^{2}+r^{2} d x^{2},
$$

where $H$ can be obtained form the momentum constraint,

$$
r^{2} H^{\prime \prime}-z r H^{\prime}+\frac{1-z}{\lambda-1} H=0 .
$$

This is the Euler equation, and has the general solution

$$
H=H_{0} r^{\sigma_{1}+\sigma_{2}}+H_{1} r^{\sigma_{1}-\sigma_{2}},
$$

where $H_{0}$ and $H_{1}$ are two integration constants, and

$$
\sigma_{1} \equiv \frac{z+1}{2}, \quad \sigma_{2} \equiv \frac{\sqrt{(z+1)^{2}+\frac{4(z-1)}{\lambda-1}}}{2} .
$$

Inserting the above expressions into eq. (3.6), we find that

$$
\begin{gathered}
\alpha_{1} H_{0}^{2} r^{2\left(\sigma_{1}+\sigma_{2}-1\right)}+\alpha_{2} H_{1}^{2} r^{2\left(\sigma_{1}-\sigma_{2}-1\right)} \\
\quad+\alpha_{3} H_{0} H_{1} r^{2\left(\sigma_{1}-1\right)}+\alpha_{4} r^{2 z}=0
\end{gathered}
$$

where

$$
\begin{aligned}
\alpha_{1} & =\frac{1}{2}(1-\lambda)\left(\sigma_{1}+\sigma_{2}\right)^{2}-\left(\sigma_{1}+\sigma_{2}-1\right), \\
\alpha_{2} & =\frac{1}{2}(1-\lambda)\left(\sigma_{1}-\sigma_{2}\right)^{2}-\left(\sigma_{1}-\sigma_{2}-1\right), \\
\alpha_{3} & =(1-\lambda)\left(\sigma_{1}^{2}-\sigma_{2}^{2}\right)-2\left(\sigma_{1}-1\right), \\
\alpha_{4} & =\Lambda-\frac{\beta z^{2}}{2}-\gamma_{1} z .
\end{aligned}
$$

Therefore, there are four possibilities, depending on the values of the constants $H_{0}$ and $H_{1}$. 
Case B.2.1) $\boldsymbol{H}_{\mathbf{0}}=\boldsymbol{H}_{\mathbf{1}}=\mathbf{0}$ : in this case, eq. (4.37) yields,

$$
\Lambda=\frac{\beta}{2} z^{2}-\gamma_{1} z=\frac{\gamma_{1}^{2}\left(2 \gamma_{1}-\beta\right)}{2\left(\gamma_{1}-\beta\right)^{2}}
$$

Since now $H=N^{r}=0$, so the corresponding solution is exactly the Lifshitz space-time given by eq. (3.15).

Case B.2.2) $\boldsymbol{H}_{\mathbf{0}} \neq \mathbf{0}, \boldsymbol{H}_{\mathbf{1}}=\mathbf{0}$ : in this case, $\Lambda$ is still given by eq. (4.39), and in addition, eq. (4.37) also requires $\alpha_{1}=0$, which yeilds,

$$
\sigma_{1}+\sigma_{2}=\alpha_{ \pm}
$$

where

$$
\alpha_{+} \equiv \frac{1+\sqrt{2 \lambda-1}}{1-\lambda}=\left\{\begin{array}{ll}
2, & \lambda=1 / 2, \\
\infty, & \lambda=1, \\
<0, & \lambda>1, \\
0^{-}, & \lambda \rightarrow \infty,
\end{array} \quad, \quad \alpha_{-} \equiv \frac{2}{1+\sqrt{2 \lambda-1}}= \begin{cases}2, & \lambda=1 / 2, \\
1, & \lambda=1, \\
<1, & \lambda>1, \\
0^{+}, & \lambda \rightarrow \infty .\end{cases}\right.
$$

Then, combining it with eq. (4.36), we find that $z=z(\lambda)$ and is given by

$$
2 \alpha_{ \pm}=\sqrt{(z+1)^{2}+\frac{4(z-1)}{\lambda-1}}+(z+1) .
$$

Thus, $H$ is given by,

$$
H=H_{0} r^{\alpha_{ \pm}} .
$$

Clearly, to have real solutions, we must require $\lambda \geq 1 / 2$. The corresponding $K$ and $R$ are given by

$$
K=H_{0} \alpha_{ \pm} r^{\alpha_{ \pm}-(z+1)}, \quad R=-2,
$$

from which we find that the non-singular condition of the spacetime at $r=\infty$ requires $\alpha_{ \pm} \leq z+1$, for which the spacetime is singular at $r=0$, unless only the equality $\alpha_{ \pm}=z+1$ holds. The latter is possible only for $z=1$ and $\lambda=1 / 2$, as it can be seen from eqs. (4.41) and (4.42), for which the metric takes the form,

$$
\begin{aligned}
d s^{2}= & -r^{2} d t^{2}+\frac{1}{r^{2}}\left(d r+H_{0} r^{2} d t\right)^{2} \\
& +r^{2} d x^{2},(z=1, \lambda=1 / 2) .
\end{aligned}
$$

It is interesting to note that the above solution can be obtained from the anti-de Sitter solution,

$$
d s^{2}=-L^{-2}\left(r^{2} d \hat{\tau}^{2}+\frac{d r^{2}}{r^{2}}+r^{2} d \hat{x}^{2}\right)
$$

by the "coordinate transformation" (4.10) with $\Sigma=-H_{0} /\left[\left(1-H_{0}^{2}\right) r\right], \hat{\tau}=L^{2} \tau, \hat{x}=x / L$, where $L \equiv \sqrt{1-H_{0}^{2}}$. As mentioned above, this is not allowed by the symmetry of the theory. Therefore, the above solution represents a different spacetime in the HL theory. 
Case B.2.3) $\boldsymbol{H}_{\mathbf{0}}=\mathbf{0}, \boldsymbol{H}_{\mathbf{1}} \neq \mathbf{0}$ : in this case we must have $\alpha_{2}=0=\alpha_{4}$. The latter yeilds eq. (4.39), while the former $\alpha_{2}=0$ yields,

$$
\sigma_{1}-\sigma_{2}=\alpha_{ \pm}
$$

where $\alpha_{ \pm}$are given by eq. (4.41). Then, the function $H$ is also given by eq. (4.43) with $H_{0}$ being replaced by $H_{1}$. As a result, the solutions are identical to the ones obtained in the last case.

Case B.2.4) $\boldsymbol{H}_{\mathbf{0}} \boldsymbol{H}_{\mathbf{1}} \neq \mathbf{0}$ : in this case, once again we find that $\Lambda$ is given by eq. (4.39). In addition, we must also have $\alpha_{1}=\alpha_{2}=\alpha_{3}=0$, which yields,

$$
\sigma_{1}=\alpha_{ \pm}, \quad \sigma_{2}=0
$$

This in turn gives

$$
H=N^{r}=\left(H_{0}+H_{1}\right) r^{\alpha_{ \pm}} .
$$

Therefore, in this case the soltuions are also the same as these given in Case B.2.2).

\subsubsection{Solutions with $W \neq 0, z$ and $\beta=0$}

In this case, from eq. (4.25) we find that

$$
r^{z-1} g f=c_{1},
$$

where $c_{1}$ is an integration constant. Then, the momentum constraint (3.4) and the dynamical equation (3.6) imply

$$
\begin{aligned}
H & =H_{0} r^{2}, & N^{r} & =c_{0} r \sqrt{r^{2}-M}, \\
f & =\frac{f_{0} \sqrt{r^{2}-M}}{r^{z}}, & g & =\frac{g_{0} r}{\sqrt{r^{2}-M}},
\end{aligned}
$$

where $c_{0} \equiv H_{0} / g_{0}, c_{1} \equiv f_{0} g_{0}$, and $f_{0}$ and $g_{0}$ are other two constants. Thus, the corresponding metric takes the form,

$$
d s^{2}=L^{2}\left\{-\left(r^{2}-M\right) d t^{2}+\frac{\left(d r+c_{0} r \sqrt{r^{2}-M} d t\right)^{2}}{r^{2}-M}+r^{2} d x^{2}\right\},
$$

where $L \equiv g_{0}$. Note that in writing the above metric, we had set $f_{0}=L$ by rescaling $t$.

The corresponding $K$ and $R$ are given by

$$
K=\frac{2 c_{0}}{L}, \quad R=-\frac{2}{L^{2}}
$$

from which we find that the spacetime is not singular at any point, including $r=M$. From the above analysis, it can be shown that this class of solutions can be also obtained from the generalized BTZ solutions (3.98) by the "illegal" coordinate transformation (4.10). 


\subsubsection{Solutions with $W \neq 0, z$ and $\lambda=1 / 2$}

When $\lambda=1 / 2$, the corresponding theory has conformal symmetry. In this particular case, if we take

$$
H=H_{0} r^{2},
$$

where $H_{0}$ is a constant, then we find that eq. (3.4) holds identically, and

$$
\begin{aligned}
(1-\lambda)\left(H^{\prime}\right)^{2}-2 H\left(\frac{H}{r}\right)^{\prime} & =0, \\
(1-\lambda) g\left[\frac{\left(H^{\prime}\right)^{2}}{2 r^{z} g f}-H\left(\frac{H^{\prime}}{r^{z} g f}\right)^{\prime}\right]-\frac{H\left(r^{z-2} g f H\right)^{\prime}}{r^{2 z-1} g f^{2}} & =0 .
\end{aligned}
$$

Then, it can be shown that the contributions of the parts involved with $H$ in eqs. (3.3) and (3.6) are zero. As a result, the functions $f$ and $g$ satisfy the same equations as in the case $H=0$, i.e., eqs. (3.8) and (3.9). Hence, any solution $f$ and $g$ found in section IV with $H=0$ is also a solution of the current case with $H$ being given by eq. (4.54). Thus, we have the following theorem.

Theorem. If $(f, g)=\left(f^{*}, g^{*}\right)$ is a solution of the field equations (3.8) and (3.9), then

$$
\left(f, g, N^{r}\right)=\left(f^{*}(r), g^{*}(r), \frac{H_{0} r^{2}}{g^{*}(r)}\right)
$$

is a solutions of eqs. (3.3), (3.4) and (3.6) with $\lambda=1 / 2$. In terms of $f^{*}$ and $g^{*}$, the metric takes the form,

$$
d s^{2}=r^{2 z} f^{*}(r)^{2} d t^{2}+\frac{g^{*}(r)^{2}}{r^{2}}\left(d r+\frac{H_{0} r^{2}}{g^{*}(r)} d t\right)^{2}+r^{2} d x^{2},
$$

for which we find that

$$
K=\frac{2 H_{0} r}{N^{*} g^{*}}, \quad R=\frac{2\left[r\left(g^{*}\right)^{\prime}-g^{*}\right]}{\left(g^{*}\right)^{3}},
$$

where $N^{*} \equiv r^{z} f^{*}$. For each of the solution $\left(f^{*}, g^{*}\right)$ given in the last section, we can analyze the global structure of the corresponding spacetime given by the metric (4.58).

Following what we did above, such studies are quite strainghtforward. So, in the following we shall not consider them, but simply note that conformal symmetry plays an important role in the AdS/CFT correspondence, and this class of solutions deserves particular attention.

\section{Conclusions}

In this paper, we have studied static vacuum solutions of quantum gravity at a Lifshitz point, proposed recently by Hořava [53], using the anisotropic scaling between time and space (1.2). The same scaling was also used in [13] to construct the Lifshitz spacetimes (1.1) in the content of the non-relativistic gauge/gravity duality. Because of this same scaling, 
lately it was argued [60] that the HL gravity should provide a minimal holographic dual for non-relativistic Lifshitz-type field theories.

In this paper, we have provided further evidences to support such a speculation. In particular, in section III we have found all the static vacuum diagonal $\left(g_{t r}=0\right)$ solutions of the HL gravity, and shown that the corresponding spacetimes have very rich structures. They can represent the generalized BTZ black holes, Lifshitz spacetimes and Lifshitz solitons, depending on the choice of the free parameters involved in the solutions [cf. figures 1-5].

In section IV, we have generalized our studies presented in section III to the nondiagonal case where $g_{t r} \neq 0$ (or $N^{r} \neq 0$ ), and found several classes of exact solutions. We have shown that there exist similar space-time structures as those found in the diagonal case.

Note that some solutions presented in sections III and IV represent incomplete spacetime, and extensions beyond certain horizons are needed. After the extension, they may represent Lifshitz black holes [28-49]. It would be very interesting to study those spacetimes in terms of the universal horizons [85-90]. In addition, Penrose's notion of conformal infinity of spacetime was generalized to the case with anisotropic scaling [61], and one would wonder how one can define black holes in terms of anisotropic conformal infinities? Further more, what is the corresponding thermodynamics of such defined black holes? Clearly, such studies are out of scope of the current paper, and we would like very much to come back to these important issues soon in another occasion.

Finally, we note that, although our studies presented in this paper have been restricted to $(2+1)$-dimensional spacetimes, we find that static vacuum solutions of the HL gravity in higher dimensional space-times exhibit similar space-time structures [91]. This is not difficult to understand, if we note that the higher dimensional space-time $d s_{D+1}^{2}$ is simply the superposition of the (2+1)-dimensional space-time given in this paper, and a $(D-2)$ spatial partner,

$$
\begin{aligned}
d s_{D+1}^{2} & =d s_{2+1}^{2} \oplus d s_{D-2}^{2} \\
& =-f^{2}(r) r^{2 z} d t^{2}+\frac{g^{2}(r)}{r^{2}}\left(d r+N^{r}(r) d t\right)^{2}+r^{2} d x^{2}+r^{2} \sum_{i=1}^{D-2} d x^{i} d x^{i}
\end{aligned}
$$

Therefore, the space-time structures are mainly determined by the sector $g_{a b} d x^{a} d x^{b}$ $(a, b=t, r)$.

With these exact vacuum solutions, it is expected that the studies of the non-relativistic Lifshitz-type gauge/gravity duality will be simplified considerably, and we wish to return to these issues soon.

\section{Acknowledgments}

This work is supported in part by DOE Grant, DE-FG02-10ER41692 (AW); Ciência Sem Fronteiras, No. 004/2013 - DRI/CAPES (AW); NSFC No. 11375153 (AW), No. 11173021 (AW), No. 11005165 (FWS), No. 11178018 (KL), No. 11075224 (KL); FAPESP No. 2012/08934-0 (KL); and NSFC No. 11205133 (QW). 


\section{A Functions $F_{V}, F^{i j}$ and $F_{a}^{i j}$}

The function $F_{V}$ presented in eq. (2.9) is given by

$$
\begin{aligned}
F_{V}= & -\beta\left(2 a_{i}^{i}+a_{i} a^{i}\right)-\frac{\beta_{1}}{\zeta^{2}}\left[3\left(a_{i} a^{i}\right)^{2}+4 \nabla_{i}\left(a_{k} a^{k} a^{i}\right)\right]+\frac{\beta_{2}}{\zeta^{2}}\left[\left(a_{i}^{i}\right)^{2}+\frac{2}{N} \nabla^{2}\left(N a_{k}^{k}\right)\right] \\
& -\frac{\beta_{3}}{\zeta^{2}}\left[\left(a_{i} a^{i}\right) a_{j}^{j}+2 \nabla_{i}\left(a_{j}^{j} a^{i}\right)-\frac{1}{N} \nabla^{2}\left(N a_{i} a^{i}\right)\right]+\frac{\beta_{4}}{\zeta^{2}}\left[a_{i j} a^{i j}+\frac{2}{N} \nabla_{j} \nabla_{i}\left(N a^{i j}\right)\right] \\
& -\frac{\beta_{5}}{\zeta^{2}}\left[R\left(a_{i} a^{i}\right)+2 \nabla_{i}\left(R a^{i}\right)\right]+\frac{\beta_{6}}{\zeta^{2}}\left[R a_{i}^{i}+\frac{1}{N} \nabla^{2}(N R)\right],
\end{aligned}
$$

The functions $\left(F_{n}\right)_{i j}$ and $\left(F_{s}^{a}\right)_{i j}$, defined in eq. (2.15), are given, respectively, by

$$
\begin{aligned}
& \left(F_{0}\right)_{i j}=-\frac{1}{2} g_{i j}, \\
& \left(F_{1}\right)_{i j}=R_{i j}-\frac{1}{2} R g_{i j}+\frac{1}{N}\left(g_{i j} \nabla^{2} N-\nabla_{j} \nabla_{i} N\right), \\
& \left(F_{2}\right)_{i j}=-\frac{1}{2} g_{i j} R^{2}+2 R R_{i j}+\frac{2}{N}\left[g_{i j} \nabla^{2}(N R)-\nabla_{j} \nabla_{i}(N R)\right], \\
& \left(F_{0}^{a}\right)_{i j}=-\frac{1}{2} g_{i j} a^{k} a_{k}+a_{i} a_{j}, \\
& \left(F_{1}^{a}\right)_{i j}=-\frac{1}{2} g_{i j}\left(a_{k} a^{k}\right)^{2}+2\left(a_{k} a^{k}\right) a_{i} a_{j}, \\
& \left.\left(F_{2}^{a}\right)_{i j}=-\frac{1}{2} g_{i j}\left(a_{k}{ }^{k}\right)^{2}+2 a_{k}{ }^{k} a_{i j}-\frac{1}{N}\left[2 \nabla_{(i}\left(N a_{j}\right) a_{k}{ }^{k}\right)-g_{i j} \nabla^{l}\left(a_{l} N a_{k}{ }^{k}\right)\right], \\
& \left(F_{3}^{a}\right)_{i j}=-\frac{1}{2} g_{i j}\left(a_{k} a^{k}\right) a_{l}{ }^{l}+a^{k}{ }_{k} a_{i} a_{j}+a_{k} a^{k} a_{i j}-\frac{1}{N}\left[\nabla_{(i}\left(N a_{j} a_{k} a^{k}\right)-\frac{1}{2} g_{i j} \nabla^{l}\left(a_{l} N a_{k} a^{k}\right)\right], \\
& \left(F_{4}^{a}\right)_{i j}=-\frac{1}{2} g_{i j} a^{m n} a_{m n}+2 a^{k}{ }_{i} a_{k j}-\frac{1}{N}\left[\nabla^{k}\left(2 N a_{(i} a_{j) k}-N a_{i j} a_{k}\right)\right], \\
& \left(F_{5}^{a}\right)_{i j}=-\frac{1}{2} g_{i j}\left(a_{k} a^{k}\right) R+a_{i} a_{j} R+a^{k} a_{k} R_{i j}+\frac{1}{N}\left[g_{i j} \nabla^{2}\left(N a_{k} a^{k}\right)-\nabla_{i} \nabla_{j}\left(N a_{k} a^{k}\right)\right], \\
& \left(F_{6}^{a}\right)_{i j}=-\frac{1}{2} g_{i j} R a_{k}{ }^{k}+a_{k}^{k} R_{i j}+R a_{i j}+\frac{1}{N}\left[g_{i j} \nabla^{2}\left(N a_{k}^{k}\right)-\nabla_{i} \nabla_{j}\left(N a_{k}{ }^{k}\right)\right.
\end{aligned}
$$

Open Access. This article is distributed under the terms of the Creative Commons Attribution License (CC-BY 4.0), which permits any use, distribution and reproduction in any medium, provided the original author(s) and source are credited.

\section{References}

[1] J. Cardy, Scaling and renormalization in statistical physics, Cambridge University Press, Cambridge U.K. (2002).

[2] S. Sachdev, Quantum phase transitions, second edition, Cambridge University Press, Cambridge U.K. (2013). 
[3] O. Aharony, S.S. Gubser, J. Maldacena, H. Ooguri and Y. Oz, Large N field theories, string theory and gravity, Phys. Rept. 323 (2000) 183 [hep-th/9905111] [INSPIRE].

[4] J. Maldacena, The gauge/gravity duality, arXiv:1106.6073 [INSPIRE].

[5] J. Polchinski, Introduction to gauge/gravity duality, arXiv:1010.6134 [INSPIRE].

[6] J.M. Maldacena, The large- $N$ limit of superconformal field theories and supergravity, Adv. Theor. Math. Phys. 2 (1998) 231 [Int. J. Theor. Phys. 38 (1999) 1113] [hep-th/9711200] [INSPIRE].

[7] S. Gubser, I.R. Klebanov and A.M. Polyakov, Gauge theory correlators from noncritical string theory, Phys. Lett. B 428 (1998) 105 [hep-th/9802109] [INSPIRE].

[8] E. Witten, Anti-de Sitter space and holography, Adv. Theor. Math. Phys. 2 (1998) 253 [hep-th/9802150] [INSPIRE].

[9] S.A. Hartnoll, Lectures on holographic methods for condensed matter physics, Class. Quant. Grav. 26 (2009) 224002 [arXiv:0903.3246] [INSPIRE].

[10] J. McGreevy, Holographic duality with a view toward many-body physics, Adv. High Energy Phys. 2010 (2010) 723105 [arXiv:0909.0518] [INSPIRE].

[11] G.T. Horowitz, Introduction to holographic superconductors, Lect. Notes Phys. 828 (2011) 313 [arXiv: 1002.1722] [inSPIRE].

[12] S. Sachdev, What can gauge-gravity duality teach us about condensed matter physics?, Ann. Rev. Condens. Matter Phys. 3 (2012) 9.

[13] S. Kachru, X. Liu and M. Mulligan, Gravity duals of Lifshitz-like fixed points, Phys. Rev. D 78 (2008) 106005 [arXiv:0808.1725] [INSPIRE].

[14] D.T. Son, Toward an AdS/cold atoms correspondence: a geometric realization of the Schrödinger symmetry, Phys. Rev. D 78 (2008) 046003 [arXiv: 0804.3972] [InSPIRE].

[15] K. Balasubramanian and J. McGreevy, Gravity duals for non-relativistic CFTs, Phys. Rev. Lett. 101 (2008) 061601 [arXiv:0804.4053] [INSPIRE].

[16] M. Taylor, Non-relativistic holography, arXiv:0812.0530 [INSPIRE].

[17] K. Balasubramanian and K. Narayan, Lifshitz spacetimes from AdS null and cosmological solutions, JHEP 08 (2010) 014 [arXiv: 1005.3291] [INSPIRE].

[18] A. Donos and J.P. Gauntlett, Lifshitz solutions of $D=10$ and $D=11$ supergravity, JHEP 12 (2010) 002 [arXiv: 1008.2062] [INSPIRE].

[19] R. Gregory, S.L. Parameswaran, G. Tasinato and I. Zavala, Lifshitz solutions in supergravity and string theory, JHEP 12 (2010) 047 [arXiv: 1009.3445] [INSPIRE].

[20] P. Dey and S. Roy, From AdS to Schrödinger/Lifshitz dual space-times without or with hyperscaling violation, JHEP 11 (2013) 113 [arXiv:1306.1071] [INSPIRE].

[21] K. Copsey and R. Mann, Pathologies in asymptotically Lifshitz spacetimes, JHEP 03 (2011) 039 [arXiv: 1011.3502] [INSPIRE].

[22] G.T. Horowitz and B. Way, Lifshitz singularities, Phys. Rev. D 85 (2012) 046008 [arXiv:1111.1243] [INSPIRE].

[23] N. Bao, X. Dong, S. Harrison and E. Silverstein, The benefits of stress: resolution of the Lifshitz singularity, Phys. Rev. D 86 (2012) 106008 [arXiv:1207.0171] [INSPIRE]. 
[24] T. Biswas, E. Gerwick, T. Koivisto and A. Mazumdar, Towards singularity and ghost free theories of gravity, Phys. Rev. Lett. 108 (2012) 031101 [arXiv:1110.5249] [INSPIRE].

[25] S. Harrison, S. Kachru and H. Wang, Resolving Lifshitz horizons, JHEP 02 (2014) 085 [arXiv:1202.6635] [INSPIRE].

[26] G. Knodel and J.T. Liu, Higher derivative corrections to Lifshitz backgrounds, JHEP 10 (2013) 002 [arXiv: 1305.3279] [inSPIRE].

[27] S. Kachru, N. Kundu, A. Saha, R. Samanta and S.P. Trivedi, Interpolating from Bianchi attractors to Lifshitz and AdS spacetimes, JHEP 03 (2014) 074 [arXiv:1310.5740] [INSPIRE].

[28] R.B. Mann, Lifshitz topological black holes, JHEP 06 (2009) 075 [arXiv:0905.1136] [INSPIRE].

[29] G. Bertoldi, B.A. Burrington and A. Peet, Black holes in asymptotically Lifshitz spacetimes with arbitrary critical exponent, Phys. Rev. D 80 (2009) 126003 [arXiv:0905.3183] [INSPIRE].

[30] K. Balasubramanian and J. McGreevy, An analytic Lifshitz black hole, Phys. Rev. D 80 (2009) 104039 [arXiv:0909.0263] [inSPIRE].

[31] E. Ayon-Beato, A. Garbarz, G. Giribet and M. Hassaine, Lifshitz black hole in three dimensions, Phys. Rev. D 80 (2009) 104029 [arXiv:0909.1347] [INSPIRE].

[32] R.-G. Cai, Y. Liu and Y.-W. Sun, A Lifshitz black hole in four dimensional $R^{2}$ gravity, JHEP 10 (2009) 080 [arXiv:0909.2807] [InSPIRE].

[33] M. Setare and D. Momeni, Plane symmetric solutions in Hor̆ava-Lifshitz theory, Int. J. Mod. Phys. D 19 (2010) 2079 [arXiv:0911.1877] [INSPIRE].

[34] Y.S. Myung, Lifshitz black holes in the Hořava-Lifshitz gravity, Phys. Lett. B 690 (2010) 534 [arXiv: 1002.4448] [INSPIRE].

[35] D.-W. Pang, On charged Lifshitz black holes, JHEP 01 (2010) 116 [arXiv:0911.2777] [INSPIRE].

[36] E. Ayon-Beato, A. Garbarz, G. Giribet and M. Hassaine, Analytic Lifshitz black holes in higher dimensions, JHEP 04 (2010) 030 [arXiv: 1001.2361] [INSPIRE].

[37] M. Dehghani and R.B. Mann, Lovelock-Lifshitz black holes, JHEP 07 (2010) 019 [arXiv: 1004.4397] [INSPIRE].

[38] M. Dehghani, R. Mann and R. Pourhasan, Charged Lifshitz black holes, Phys. Rev. D 84 (2011) 046002 [arXiv: 1102.0578] [INSPIRE].

[39] W. Brenna, M. Dehghani and R. Mann, Quasi-topological Lifshitz black holes, Phys. Rev. D 84 (2011) 024012 [arXiv:1101.3476] [INSPIRE].

[40] J. Matulich and R. Troncoso, Asymptotically Lifshitz wormholes and black holes for Lovelock gravity in vacuum, JHEP 10 (2011) 118 [arXiv:1107.5568] [INSPIRE].

[41] I. Amado and A.F. Faedo, Lifshitz black holes in string theory, JHEP 07 (2011) 004 [arXiv:1105.4862] [INSPIRE].

[42] L. Barclay, R. Gregory, S. Parameswaran, G. Tasinato and I. Zavala, Lifshitz black holes in IIA supergravity, JHEP 05 (2012) 122 [arXiv:1203.0576] [INSPIRE]. 
[43] H. Lü, Y. Pang, C. Pope and J.F. Vazquez-Poritz, AdS and Lifshitz black holes in conformal and Einstein-Weyl gravities, Phys. Rev. D 86 (2012) 044011 [arXiv:1204.1062] [InSPIRE].

[44] S.H. Hendi and B. Eslam Panah, Asymptotically Lifshitz black hole solutions in $F(R)$ gravity, Can. J. Phys. 92 (2013) 1 [arXiv:1309.2135] [INSPIRE].

[45] M.-I. Park, The rotating black hole in renormalizable quantum gravity: the three-dimensional Hoŕava gravity case, Phys. Lett. B 718 (2013) 1137 [arXiv:1207.4073] [INSPIRE].

[46] M. Gutperle, E. Hijano and J. Samani, Lifshitz black holes in higher spin gravity,

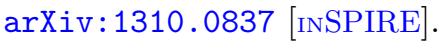

[47] H.-S. Liu and H. Lu, Lifshitz and Schrödinger vacua, superstar resolution in gauged maximal supergravities, JHEP 02 (2014) 122 [arXiv:1310.8348] [INSPIRE].

[48] M. Bravo-Gaete and M. Hassaine, Lifshitz black holes with arbitrary dynamical exponent in Horndeski theory, arXiv:1312.7736 [INSPIRE].

[49] D.O. Devecioglu, Lifshitz black holes in Einstein-Yang-Mills theory, arXiv:1401.2133 [INSPIRE].

[50] U.H. Danielsson and L. Thorlacius, Black holes in asymptotically Lifshitz spacetime, JHEP 03 (2009) 070 [arXiv: 0812.5088] [INSPIRE].

[51] R. Mann, L. Pegoraro and M. Oltean, Lifshitz solitons, Phys. Rev. D 84 (2011) 124047 [arXiv: 1109.5044] [INSPIRE].

[52] H.A. Gonzalez, D. Tempo and R. Troncoso, Field theories with anisotropic scaling in 2D, solitons and the microscopic entropy of asymptotically Lifshitz black holes, JHEP 11 (2011) 066 [arXiv: 1107.3647] [INSPIRE].

[53] P. Hořava, Quantum gravity at a Lifshitz point, Phys. Rev. D 79 (2009) 084008 [arXiv:0901.3775] [INSPIRE].

[54] D. Blas, O. Pujolàs and S. Sibiryakov, Models of non-relativistic quantum gravity: the good, the bad and the healthy, JHEP 04 (2011) 018 [arXiv: 1007.3503] [INSPIRE].

[55] S. Mukohyama, Hor̆ava-Lifshitz cosmology: a review, Class. Quant. Grav. 27 (2010) 223101 [arXiv: 1007.5199] [INSPIRE].

[56] P. Hořava, General covariance in gravity at a Lifshitz point, Class. Quant. Grav. 28 (2011) 114012 [arXiv: 1101.1081] [INSPIRE].

[57] T. Clifton, P.G. Ferreira, A. Padilla and C. Skordis, Modified gravity and cosmology, Phys. Rept. 513 (2012) 1 [arXiv:1106.2476] [INSPIRE].

[58] T. Pavlopoulos, Breakdown of Lorentz invariance, Phys. Rev. 159 (1967) 1106 [InSPIRE].

[59] S. Chadha and H.B. Nielsen, Lorentz invariance as a low-energy phenomenon, Nucl. Phys. B 217 (1983) 125 [INSPIRE].

[60] T. Griffin, P. Hořava and C.M. Melby-Thompson, Lifshitz gravity for Lifshitz holography, Phys. Rev. Lett. 110 (2013) 081602 [arXiv:1211.4872] [InSPIRE].

[61] P. Hořava and C.M. Melby-Thompson, Anisotropic conformal infinity, Gen. Rel. Grav. 43 (2011) 1391 [arXiv: 0909.3841] [INSPIRE].

[62] A. Wang, Stationary and slowly rotating spacetimes in Horava-Lifshitz gravity, Phys. Rev. Lett. 110 (2013) 091101 [arXiv:1212.1876] [INSPIRE]. 
[63] A. Borzou, K. Lin and A. Wang, Static electromagnetic fields and charged black holes in general covariant theory of Hořava-Lifshitz gravity, JCAP 02 (2012) 025 [arXiv:1110.1636] [INSPIRE].

[64] J. Greenwald, J. Lenells, J. Lu, V. Satheeshkumar and A. Wang, Black holes and global structures of spherical spacetimes in Horava-Lifshitz theory, Phys. Rev. D 84 (2011) 084040 [arXiv:1105.4259] [INSPIRE].

[65] E.B. Kiritsis and G. Kofinas, On Hořava-Lifshitz 'black holes', JHEP 01 (2010) 122 [arXiv:0910.5487] [INSPIRE].

[66] T. Suyama, Notes on matter in Hořava-Lifshitz gravity, JHEP 01 (2010) 093 [arXiv: 0909.4833] [INSPIRE].

[67] D. Capasso and A.P. Polychronakos, Particle kinematics in Hořava-Lifshitz gravity, JHEP 02 (2010) 068 [arXiv:0909.5405] [INSPIRE].

[68] J. Alexandre, K. Farakos, P. Pasipoularides and A. Tsapalis, Schwinger-Dyson approach for a Lifshitz-type Yukawa model, Phys. Rev. D 81 (2010) 045002 [arXiv:0909.3719] [INSPIRE].

[69] J.M. Romero, V. Cuesta, J.A. Garcia and J.D. Vergara, Conformal anisotropic mechanics and the Horava dispersion relation, Phys. Rev. D 81 (2010) 065013 [arXiv:0909.3540] [INSPIRE].

[70] S.K. Rama, Particle motion with Hor̆ava-Lifshitz type dispersion relations, arXiv:0910.0411 [INSPIRE].

[71] L. Sindoni, A note on particle kinematics in Hor̆ava-Lifshitz scenarios, arXiv:0910.1329 [INSPIRE].

[72] S.W. Hawking and G.F.R. Ellis, The large scale structure of space-time, Cambridge Monographs on Mathematical Physics, Cambridge University Press, Cambridge U.K. (1973).

[73] F.J. Tipler, Black holes in closed universes, Nature 270 (1977) 500.

[74] S. Hayward, General laws of black hole dynamics, Phys. Rev. D 49 (1994) 6467 [InSPIRE].

[75] S.A. Hayward, Gravitational waves, black holes and cosmic strings in cylindrical symmetry, Class. Quant. Grav. 17 (2000) 1749 [gr-qc/9909070] [INSPIRE].

[76] A. Wang, Critical collapse of cylindrically symmetric scalar field in four-dimensional Einstein's theory of gravity, Phys. Rev. D 68 (2003) 064006 [gr-qc/0307071] [INSPIRE].

[77] A. Wang, Comment on 'Absence of trapped surfaces and singularities in cylindrical collapse', Phys. Rev. D 72 (2005) 108501 [gr-qc/0309003] [inSPIRE].

[78] A. Wang, No-go theorem in spacetimes with two commuting spacelike Killing vectors, Gen. Rel. Grav. 37 (2005) 1919 [INSPIRE].

[79] A.Y. Miguelote, N. Tomimura and A. Wang, Gravitational collapse of selfsimilar perfect fluid in $2+1$ gravity, Gen. Rel. Grav. 36 (2004) 1883 [gr-qc/0304035] [INSPIRE].

[80] P. Sharma, A. Tziolas, A. Wang and Z.-C. Wu, Spacetime singularities in string and its low dimensional effective theory, Int. J. Mod. Phys. A 26 (2011) 273 [arXiv:0901.2676] [INSPIRE].

[81] J.W. Elliott, G.D. Moore and H. Stoica, Constraining the new Aether: Gravitational Cerenkov radiation, JHEP 08 (2005) 066 [hep-ph/0505211] [INSPIRE]. 
[82] G.D. Moore and A.E. Nelson, Lower bound on the propagation speed of gravity from gravitational Cherenkov radiation, JHEP 09 (2001) 023 [hep-ph/0106220] [INSPIRE].

[83] M. Pospelov and Y. Shang, On Lorentz violation in Hor̆ava-Lifshitz type theories, Phys. Rev. D 85 (2012) 105001 [arXiv: 1010.5249] [INSPIRE].

[84] M. Pospelov and C. Tamarit, Lifshitz-sector mediated SUSY breaking, JHEP 01 (2014) 048 [arXiv:1309.5569] [INSPIRE].

[85] D. Blas and S. Sibiryakov, Hořava gravity versus thermodynamics: The Black hole case, Phys. Rev. D 84 (2011) 124043 [arXiv:1110.2195] [InSPIRE].

[86] P. Berglund, J. Bhattacharyya and D. Mattingly, Mechanics of universal horizons, Phys. Rev. D 85 (2012) 124019 [arXiv: 1202.4497] [INSPIRE].

[87] P. Berglund, J. Bhattacharyya and D. Mattingly, Thermodynamics of universal horizons in Einstein-aether theory, Phys. Rev. Lett. 110 (2013) 071301 [arXiv:1210.4940] [INSPIRE].

[88] B. Cropp, S. Liberati and M. Visser, Surface gravities for non-Killing horizons, Class. Quant. Grav. 30 (2013) 125001 [arXiv:1302.2383] [InSPIRE].

[89] M. Saravani, N. Afshordi and R.B. Mann, Dynamical emergence of universal horizons during the formation of black holes, arXiv:1310.4143 [INSPIRE].

[90] B. Cropp, S. Liberati, A. Mohd and M. Visser, Ray tracing Einstein-aether black holes: universal versus Killing horizons, Phys. Rev. D 89 (2014) 064061 [arXiv:1312.0405] [INSPIRE].

[91] K. Lin, F.-W. Shu, A. Wang and Q. Wu, in preparation.

[92] R. Arnowitt, S. Deser and C.W. Misner, Republication of: the dynamics of general relativity, Gen. Rel. Grav. 40 (2008) 1997.

[93] C.W. Misner, K.S. Thorne and J.A. Wheeler, Gravitation, W.H. Freeman and Company, San Francisco, U.S.A. (1973).

[94] S. Carlip, Quantum gravity in $2+1$ dimensions, Cambridge Monographs on Mathematical Physics, Cambridge University Press, Cambridge U.K. (2003).

[95] T. Zhu, Q. Wu, A. Wang and F.-W. Shu, U(1) symmetry and elimination of spin-0 gravitons in Hoŕava-Lifshitz gravity without the projectability condition, Phys. Rev. D 84 (2011) 101502 [arXiv:1108.1237] [INSPIRE].

[96] T. Zhu, F.-W. Shu, Q. Wu and A. Wang, General covariant Horaava-Lifshitz gravity without projectability condition and its applications to cosmology, Phys. Rev. D 85 (2012) 044053 [arXiv:1110.5106] [INSPIRE].

[97] K. Lin, S. Mukohyama, A. Wang and T. Zhu, Post-Newtonian approximations in the Hor̆ava-Lifshitz gravity with extra U(1) symmetry, arXiv:1310.6666 [INSPIRE].

[98] M. Bañados, C. Teitelboim and J. Zanelli, The black hole in three-dimensional space-time, Phys. Rev. Lett. 69 (1992) 1849 [hep-th/9204099] [INSPIRE].

[99] R.-G. Cai and A. Wang, Singularities in Hořava-Lifshitz theory, Phys. Lett. B 686 (2010) 166 [arXiv: 1001.0155] [INSPIRE].

[100] P. Painleve, La mécanique classique et la théorie de la relativité, C. R. Acad. Sci. (Paris) 173 (1921) 677. 
[101] A. Gullstrand, Allgemeine Lösung des statischen Einkörperproblems in der Einsteinschen Gravitationstheorie, Arkiv. Mat. Astron. Fys. 16 (1922) 1

[102] E.M. Lifshitz, On the theory of second-order phase transitions I, Zh. Eksp. Teor. Fiz. 11 (1941) 255.

[103] E.M. Lifshitz, On the theory of second-order phase transitions II, Zh. Eksp. Teor. Fiz. 11 (1941) 269. 